\title{
Six-point remainder function in multi-Regge-kinematics: an efficient approach in momentum space
}

\author{
Johannes Broedel ${ }^{a, b}$ and Martin Sprenger ${ }^{a}$ \\ ${ }^{a}$ Institut für Theoretische Physik, Eidgenössische Technische Hochschule Zürich, \\ Wolfgang-Pauli-Strasse 27, 8093 Zürich, Switzerland \\ ${ }^{b}$ Institut für Mathematik und Institut für Physik, Humboldt-Universität zu Berlin, \\ IRIS Adlershof, Zum Großen Windkanal 6, 12489 Berlin, Germany \\ E-mail: jbroedel@physik.hu-berlin.de, sprengerm@itp.phys.ethz.ch
}

ABSTRACT: Starting from the known all-order expressions for the BFKL eigenvalue and impact factor, we establish a formalism allowing the direct calculation of the six-point remainder function in $\mathcal{N}=4$ super-Yang-Mills theory in momentum space to - in principle - all orders in perturbation theory. Based upon identities which relate different integrals contributing to the inverse Fourier-Mellin transform recursively, the formalism allows to easily access the full remainder function in multi-Regge kinematics up to 7 loops and up to 10 loops in the fourth logarithmic order. Using the formalism, we prove the all-loop formula for the leading logarithmic approximation proposed by Pennington and investigate the behavior of several newly calculated functions.

KEYWORDS: Scattering Amplitudes, Extended Supersymmetry

ARXIV EPRINT: 1512.04963 


\section{Contents}

1 Introduction 1

2 BFKL/remainder function basics 3

3 Solving the integral $\quad 8$

$\begin{array}{lll}3.1 & \text { Basic mechanism } & 10\end{array}$

$\begin{array}{lll}3.2 & \text { Integrals of the form } \mathcal{I}\left[\chi_{+}^{n_{+}} \chi_{-}^{n_{-}}\right] & 10\end{array}$

$\begin{array}{lll}3.3 & \text { Basis integrals } & 12\end{array}$

$\begin{array}{ll}3.4 \text { General integral } & 14\end{array}$

$\begin{array}{lll}3.5 & \text { Example: } \mathcal{I}\left[\left(D_{\nu}^{2} E\right) E\right] & 15\end{array}$

$\begin{array}{lll}4 & \text { NMHV } & 17\end{array}$

5 Applications $\quad 18$

$\begin{array}{lll}5.1 & \mathrm{~N}^{3} \mathrm{LLA} / \mathrm{N}^{4} \mathrm{LLA} & 18\end{array}$

$\begin{array}{ll}5.2 \text { Collinear-Regge limit } & 19\end{array}$

6 Proof of Pennington's formula 21

6.1 Pennington's formula 21

6.2 Strategy of the proof 21

6.3 Proof of the $\zeta$-free part 22

6.4 Including the $\zeta$ 's 23

$\begin{array}{llr}7 & \text { Conclusions } & 25\end{array}$

A Differential equations for insertions of $\chi_{ \pm} \quad 26$

\section{Introduction}

Starting with the seminal papers of Balitsky, Fadin, Kuraev and Lipatov (BFKL) [1-4], the study of the multi-Regge regime of scattering amplitudes in non-Abelian gauge theories has a long and rich history. Besides being of phenomenological importance, it is in this kinematical regime that integrable structures and relations to spin chain models were identified first [5-7]. A key feature of the multi-Regge regime is that the perturbative expansion is reorganized naturally: instead of expanding in the number of loops, amplitudes in the multi-Regge limit are usually expanded in logarithmic orders, starting with the leading logarithmic approximation (or LLA for short) and followed by $\mathrm{N}^{k}$ LLA (for (Next-to) ${ }^{k}$-LLA) which are subleading in kinematics. Hence, each logarithmic order contains information from all loop orders and therefore from finite coupling, albeit in a very specific way. 
Multi-Regge kinematics (MRK) is thus a natural regime to study in planar $\mathcal{N}=4$ super Yang-Mills theory, which is believed to be solvable at finite coupling, as well. Indeed, the study of the multi-Regge regime in this theory revealed the failure of the Bern-DixonSmirnov (BDS) ansatz [8] for the six-gluon MHV amplitude in refs. $[9,10]$ before the explicit two-loop calculation of the remainder function appeared in refs. [11, 12]. Further results include the calculation of the remainder function at strong coupling [13-17], the study of higher-point amplitudes [18-21] and the identification of the correct basis functions describing the multi-Regge limit [22-24]. Those functions will play a key role later on.

Direct calculations allowed the determination of the six-gluon MHV and NMHV remainder function in Fourier-Mellin space up to NLLA [25-27]. However, modern amplitude techniques such as the amplitude bootstrap in ref. [28] allowed to push the known results up to $\mathrm{N}^{2} \mathrm{LLA}$ (and $\mathrm{N}^{3} \mathrm{LLA}$ for the impact factor). The efforts to determine the remainder function to increasing accuracy culminated in a recent paper [29] in which conjectures for exact expressions in the multi-Regge regime are put forward. These expressions are derived by an analytic continuation from the collinear regime which is described by the Wilson loop OPE [30-33] and reproduce the known data at weak and strong coupling.

These equations and their solutions are, however, formulated in Fourier-Mellin space. The calculation of the remainder function in momentum space therefore still requires carrying out an - in general - tedious inverse Fourier-Mellin transform. The problem is simplified by the observation made in [22] that all integrals appearing yield a special class of functions, namely the single-valued harmonic polylogarithms (SVHPLs) constructed in ref. [34]. This, together with symmetry considerations, allows to write down a compact ansatz for the result of each integral, which can then be matched with the first orders of the integral's series expansion. While feasible for low weights, the matching becomes cumbersome for large weights.

Another step forward was made in ref. [35]: there it was shown that the analytic continuation from the collinear regime to the multi-Regge regime can be carried out termby-term. More importantly, the authors use an observation made in ref. [36] to argue that in carrying out the inverse Fourier-Mellin transform it is sufficient to focus on a specific subset of terms. Demanding single-valuedness then restores the full answer. This method, too, is algorithmic in nature, but is not formulated in momentum space. The procedure is explained in section 2 below.

In this paper, we combine the implications of single-valuedness alluded to above with relations between different integrals: considering the residues from which the single-valued result can be obtained, we can trace back all integrals to a small set of trivial basis integrals. Based on those relations, we propose an algorithm for the calculation of the six-point remainder function at any loop order and any logarithmic order. The resulting expressions are generated directly in momentum space by operations acting on SVHPLs naturally, namely by appending and prepending indices and applying shuffles. In this sense, this paper is a natural extension of the work [23], in which all-loop formulæ for LLA in momentum space were conjectured, to all logarithmic orders. In fact, we are able to prove Pennington's formula for LLA based on our algorithm. 
This paper is organized as follows. In section 2 we review the relevant formulæ to introduce notation and describe some key features of SVHPLs. Section 3 contains an elaborate description of our formalism in the MHV case along with several examples, and is followed by a discussion of the NMHV case in section 4. Based on our algorithm, we extend the known results for $\mathrm{N}^{3}$ LLA and $\mathrm{N}^{4}$ LLA up to 10 and 9 loops, respectively, in section 5.1 and consider the collinear-Regge limit as an application of our results in section 5.2. Finally, we provide a proof of the conjectured all-loop LLA formulæ put forward in ref. [23] in section 6 before summarizing our results in section 7 .

\section{BFKL/remainder function basics}

We are interested in the six-point remainder functions $R_{6}^{\mathrm{MHV}}$ and $R_{6}^{\mathrm{NMHV}}$ of $\mathcal{N}=4$ super-Yang-Mills theory, which describe the deviation of the full amplitude from the BDS ansatz [8],

$$
\begin{aligned}
A_{6}^{\mathrm{MHV}} & =A_{\mathrm{BDS}} e^{R_{6}^{\mathrm{MHV}}}, \\
A_{6}^{\mathrm{NMHV}} & =A_{\mathrm{BDS}} R_{6}^{\mathrm{NMHV}} .
\end{aligned}
$$

Note that in defining the remainder function for NMHV we follow the conventions in ref. [37]. Switching from the external momenta $k_{i}$ to dual variables, $k_{i}=: x_{i}-x_{i+1}=: x_{i i+1}$, the multi-Regge limit of the six-gluon remainder functions is described by the following behavior of the dual conformal cross ratios:

$$
u_{1}:=\frac{x_{13}^{2} x_{46}^{2}}{x_{14}^{2} x_{36}^{2}} \rightarrow 1, \quad u_{2}:=\frac{x_{24}^{2} x_{15}^{2}}{x_{25}^{2} x_{14}^{2}} \rightarrow 0, \quad u_{3}:=\frac{x_{35}^{2} x_{26}^{2}}{x_{36}^{2} x_{25}^{2}} \rightarrow 0,
$$

such that the reduced cross ratios

$$
\tilde{u}_{2}:=\frac{u_{2}}{1-u_{1}}=: \frac{1}{|1+w|^{2}}, \quad \tilde{u}_{3}:=\frac{u_{3}}{1-u_{1}}=: \frac{|w|^{2}}{|1+w|^{2}}
$$

remain finite. As usual, we describe the kinematics with the large cross ratio $u_{1}$ and the parameters $w, w^{*}$, which parameterize the remaining kinematical freedom in the multiRegge regime. Taking the limit eq. (2.2) of the cross ratios naïvely will lead to a vanishing remainder function. However, one obtains a non-trivial result by first performing the analytic continuation

$$
u_{1} \rightarrow e^{-2 i \pi} u_{1}
$$

to the so-called Mandelstam region before taking the limit (2.2).

While the kinematical setup is universal for the six-point remainder functions, the integrals appearing in the calculation depend on the helicity configuration. Therefore the discussion in the current as well in the next section will be conducted on the example of $R_{6}^{\mathrm{MHV}}$, while the appropriate modifications in order to obtain $R_{6}^{\mathrm{NMHV}}$ are described in section 4 . 
In the Mandelstam region the six-point $\mathrm{MHV}^{1}$ remainder function in multi-Regge kinematics can be determined from the following integral $[9,25,26]$ :

$$
\begin{aligned}
\left.e^{R_{6}^{\mathrm{MHV}}+i \pi \delta}\right|_{\mathrm{MRK}}= & \cos \pi \omega_{a b}+i \frac{a}{2} \sum_{n=-\infty}^{\infty}(-1)^{n}\left(\frac{w}{w^{*}}\right)^{\frac{n}{2}} \int_{-\infty}^{\infty} \frac{\mathrm{d} \nu}{\nu^{2}+\frac{n^{2}}{4}}|w|^{2 i \nu} \Phi_{\mathrm{reg}}^{\mathrm{MHV}}(\nu, n) \\
& \times \exp \left[-\omega(\nu, n)\left(\log \left(1-u_{1}\right)+i \pi+\frac{1}{2} \log \frac{|w|^{2}}{|1+w|^{4}}\right)\right]
\end{aligned}
$$

where $a=\frac{g^{2} N_{\mathrm{c}}}{8 \pi^{2}}$. The two contributions in eq. (2.5) have a nice physical interpretation: the first term describes the contribution of a Regge pole exchange while the second term describes the contribution of a Regge cut generated by a bound state of two Reggeons. In the latter contribution the only unknown quantities are the impact factor $\Phi_{\text {reg }}^{\mathrm{MHV}}(\nu, n)$ describing the coupling of the bound state of Reggeons to the produced gluons and the BFKL eigenvalue $\omega(\nu, n)$ describing the evolution of the bound state. Both quantities have a loop expansion in powers of $a$ :

$$
\begin{aligned}
\omega(\nu, n) & =-a\left(E^{(0)}+a E^{(1)}+a^{2} E^{(2)}+\mathcal{O}\left(a^{3}\right)\right), \\
\Phi_{\mathrm{reg}}^{\mathrm{MHV}} & =1+a \Phi^{(1), \mathrm{MHV}}+a^{2} \Phi^{(2), \mathrm{MHV}}+\mathcal{O}\left(a^{3}\right) .
\end{aligned}
$$

Furthermore, $\delta$ corresponds to a phase which in the full amplitude eq. (2.1a) cancels with a similar contribution arising from the BDS ansatz [8]. Both $\delta$ and the Regge pole contribution $\omega_{a b}$ are related to the cusp anomalous dimension $\gamma_{K}$ via

$$
\omega_{a b}=\frac{1}{8} \gamma_{K}(a) \log |w|^{2} \quad \text { and } \quad \delta=\frac{1}{8} \gamma_{K}(a) \log \frac{|w|^{2}}{|1+w|^{4}}
$$

and are therefore known to all orders in perturbation theory [38].

Due to the behavior of the cross ratio $u_{1}$ in the multi-Regge limit eq. (2.2), the expression $\log \left(1-u_{1}\right)$ in the integrand of eq. (2.5) is large and the remainder function can be conveniently organized in powers of this logarithm at each loop order. In particular,

$$
\left.R_{6}^{\mathrm{MHV}}\right|_{\mathrm{MRK}}=2 \pi i \sum_{\ell=2}^{\infty} \sum_{n=0}^{\ell-1} a^{\ell} \log ^{n}\left(1-u_{1}\right)\left[g_{n}^{(\ell)}\left(w, w^{*}\right)+2 \pi i h_{n}^{(\ell)}\left(w, w^{*}\right)\right] .
$$

In eq. (2.8), all terms with $n=\ell-1$ are referred to as the leading logarithmic approximation (LLA) and similarly the terms with $n=\ell-1-k$ are called (Next-to) ${ }^{k}$-LLA. Fortunately, the imaginary and real parts $g_{n}^{(\ell)}$ and $h_{n}^{(\ell)}$ are not independent [22]: all functions $h_{n}^{(\ell)}$ can be determined from the imaginary parts $g_{n}^{(\ell)}$. Thus the knowledge of all functions $g_{n}^{(\ell)}$ will pin down the remainder function.

Accordingly, the problem of calculating the remainder function $\left.R_{6}^{\mathrm{MHV}}\right|_{\mathrm{MRK}}$ via eq. (2.5) boils down to evaluating the real part of the sum over the integral. The crucial ingredients here are the loop expansions of the impact factor $\Phi_{\mathrm{reg}}^{\mathrm{MHV}}(\nu, n)$ and the BFKL eigenvalue

\footnotetext{
${ }^{1}$ Note that in all-outgoing convention this corresponds to the helicity configuration $(++-++-)$, where legs 1,2,3 and 6 are the high-energy lines.
} 
$\omega(\nu, n)$ in eq. (2.6). The first two orders of these quantities are known from direct calculation $[9,25,26]$, while one further order of the BFKL eigenvalue and two further orders for the impact factor could be determined from the amplitude bootstrap in refs. [22, 28]. A general solution, however, was found by Basso, Caron-Huot and Sever [29] only recently: the key idea is to link the solution of eq. (2.6) to quantities appearing in the Wilson loop OPE [30-33], which are known exactly from integrability, via analytic continuation. Using this method, the eigenvalue $\omega$ and impact factor $\Phi_{\text {reg }}^{\mathrm{MHV}}$ can be calculated using the solution of the BES equation [38] to arbitrary order in the coupling constant $a$ in principle.

Higher orders of the BFKL eigenvalue and the impact factor are usually expressed in terms of the quantities $N$ and $V[22]$

$$
N:=\frac{n}{\nu^{2}+\frac{|n|^{2}}{4}} \quad \text { and } \quad V:=\frac{i \nu}{\nu^{2}+\frac{|n|^{2}}{4}},
$$

the derivative

$$
D_{\nu}:=-i \partial_{\nu}
$$

and the lowest order of the BFKL eigenvalue

$$
E^{(0)}=-\frac{1}{2} \operatorname{sgn}(n) N+\psi\left(1+i \nu+\frac{|n|}{2}\right)+\psi\left(1-i \nu+\frac{|n|}{2}\right)-2 \psi(1) .
$$

In this language, the first orders of eigenvalue (beyond eq. (2.11)) and impact factor take the form

$$
\begin{aligned}
E^{(1)}= & -\frac{1}{4} D_{\nu}^{2} E^{(0)}+\frac{1}{2} V D_{\nu} E^{(0)}-\zeta_{2} E^{(0)}-3 \zeta_{3} \\
E^{(2)}= & \frac{1}{8}\left(\frac{1}{6} D_{\nu}^{4} E^{(0)}-V D_{\nu}^{3} E^{(0)}+\left(V^{2}+2 \zeta_{2}\right) D_{\nu}^{2} E^{(0)}-V\left(N^{2}+8 \zeta_{2}\right) D_{\nu} E^{(0)}\right. \\
& \left.+\zeta_{3}\left(4 V^{2}+N^{2}\right)+44 \zeta_{4} E^{(0)}+16 \zeta_{2} \zeta_{3}+80 \zeta_{5}\right), \\
\Phi^{(1), \mathrm{MHV}}= & -\frac{1}{2}\left(E^{(0)}\right)^{2}-\frac{3}{8} N^{2}-\zeta_{2}, \\
\Phi^{(2), \mathrm{MHV}}= & \frac{1}{2}\left(\Phi^{(1), \mathrm{MHV}}\right)^{2}-E^{(1)} E^{(0)}+\frac{1}{8}\left(D_{\nu} E^{(0)}\right)^{2}+\frac{5}{64} N^{2}\left(N^{2}+4 V^{2}\right) \\
& -\frac{\zeta_{2}}{4}\left(2\left(E^{(0)}\right)^{2}+N^{2}+6 V^{2}\right)+\frac{17 \zeta_{4}}{4} .
\end{aligned}
$$

Due to the structure of the integrability results of ref. [29] for the eigenvalue $\omega$ and impact factor $\Phi_{\mathrm{reg}}^{\mathrm{MHV}}$, the expansion parameters $E^{(k)}, \Phi^{(k), \mathrm{MHV}}$ can always be written as a function of $V, N$, as well as $E^{(0)}$ and derivatives thereof.

For reasons to become clear below we will later replace the quantities $N$ and $V$ with new variables $\chi_{+}$and $\chi_{-}$:

$$
\chi_{+}:=\left(V+\frac{N}{2}\right) \quad \text { and } \quad \chi_{-}:=\left(V-\frac{N}{2}\right) .
$$

As discussed in ref. [22], the functions $g_{n}^{(\ell)}$ can be expressed in terms of single-valued harmonic polylogarithms (SVHPLs) [34]. SVHPLs are linear combinations of products of 
usual harmonic polylogarithms (HPLs) in the variables $z$ and $z^{*}$, which are combined in such a way that their branch cuts cancel. Different SVHPLs ${ }^{2} \mathcal{L}_{s}(z)=\mathcal{L}_{s}$ are labeled by a word $s$ built from the alphabet $\left\{x_{0}, x_{1}\right\}$, which is reflected in the identification

$$
x_{i_{1}} \ldots x_{i_{n}} \leftrightarrow \mathcal{L}_{i_{1} \ldots i_{n}}(z)
$$

Whenever necessary, a word built from letters $x_{0}$ and $x_{1}$ is understood to be replaced by the appropriate polylogarithm using the above assignment.

As derived by Brown in ref. [34], the particular combinations of HPLs resulting in SVHPLs are unique and can be extracted from demanding triviality of the monodromies around singular points of HPLs. Nicely, this single-valuedness condition can be expressed as an equation involving the Drinfeld associator. Explicitly, the SVHPLs with lowest weights are given by

$$
\begin{aligned}
\mathcal{L}_{0}(z) & =H_{0}(z)+H_{0}\left(z^{*}\right) \\
\mathcal{L}_{1}(z) & =H_{1}(z)+H_{1}\left(z^{*}\right) \\
\mathcal{L}_{00}(z) & =H_{00}(z)+H_{00}\left(z^{*}\right)+H_{0}(z) H_{0}\left(z^{*}\right) \\
\mathcal{L}_{10}(z) & =H_{10}(z)+H_{01}\left(z^{*}\right)+H_{1}(z) H_{0}\left(z^{*}\right) \\
\mathcal{L}_{101}(z) & =H_{101}(z)+H_{101}\left(z^{*}\right)+H_{10}(z) H_{1}\left(z^{*}\right)+H_{1}(z) H_{10}\left(z^{*}\right)
\end{aligned}
$$

With increasing weight, these expressions become more complex and $\zeta$-values enter explicitly, for example

$$
\begin{aligned}
\mathcal{L}_{1010}(z)= & H_{1010}(z)+H_{0101}\left(z^{*}\right)+H_{101}(z) H_{0}\left(z^{*}\right)+H_{1}(z) H_{010}\left(z^{*}\right) \\
& +H_{10}(z) H_{01}\left(z^{*}\right)-4 \zeta_{3} H_{1}\left(z^{*}\right) .
\end{aligned}
$$

An elaborate introduction to SVHPLs in the context of the six-point remainder function in MRK can be found in section 3 of ref. [22]. Here, we will simply describe the essential properties of SVHPLs for the sections to follow. First, two SVHPLs labeled by words $s_{1}$ and $s_{2}$ satisfy the shuffle relation

$$
\mathcal{L}_{s_{1}}(z) \mathcal{L}_{s_{2}}(z)=\sum_{s \in s_{1} \uplus s_{2}} \mathcal{L}_{s}(z)
$$

where $s_{1} \amalg s_{2}$ denotes all permutations of $s_{1} \cup s_{2}$ which preserve the order of elements in $s_{1}$ and $s_{2}$. Furthermore, the SVHPLs satisfy differential equations which can be most easily stated by introducing a generating functional for the SVHPLs,

$$
\mathcal{L}(z)=\sum_{s \in X^{*}} \mathcal{L}_{s}(z) s=1+\mathcal{L}_{0}(z) x_{0}+\mathcal{L}_{1}(z) x_{1}+\mathcal{L}_{00}(z) x_{0} x_{0}+\mathcal{L}_{01}(z) x_{0} x_{1}+\ldots,
$$

\footnotetext{
${ }^{2}$ As will be clear from the examples given in the text, the $\mathcal{L}_{s}(z)$ are functions of both $z$ and $z^{*}$. It is, however, customary, to simply denote the functions $\mathcal{L}_{s}(z)$.
} 
where $X^{*}$ are all words in the alphabet $\left\{x_{0}, x_{1}\right\}$. This generating functional satisfies the differential equations

$$
\frac{\partial}{\partial z} \mathcal{L}(z)=\left(\frac{x_{0}}{z}+\frac{x_{1}}{1-z}\right) \mathcal{L}(z), \quad \frac{\partial}{\partial z^{*}} \mathcal{L}(z)=\mathcal{L}(z)\left(\frac{y_{0}}{z^{*}}+\frac{y_{1}}{1-z^{*}}\right)
$$

where $\left\{y_{0}, y_{1}\right\}$ is an additional alphabet, which appears in the construction of ref. [34] and is related by the single-valuedness condition to the alphabet $\left\{x_{0}, x_{1}\right\}$ introduced above. Solving this condition order by order, one finds

$$
\begin{aligned}
& y_{0}=x_{0} \quad \text { and } \\
& y_{1}=x_{1}-\zeta_{3}\left(2 x_{0} x_{0} x_{1} x_{1}-4 x_{0} x_{1} x_{0} x_{1}+2 x_{0} x_{1} x_{1} x_{1}+4 x_{1} x_{0} x_{1} x_{0}+\cdots\right)+\cdots,
\end{aligned}
$$

where the words appearing in the deviation of $y_{1}$ from $x_{1}$ always include at least two $x_{1}$ 's and are always multiplied by a $\zeta$-value. From eq. (2.19) we see that derivatives with respect to $z$ act by taking off the first index of a given SVHPL and multiplication with some factor. However, due to the non-trivial relation between the two alphabets, taking derivatives with respect to $z^{*}$ does not simply act by chopping off the last index, but can lead to additional terms which always include $\zeta$-values. These additional terms constitute one of the main challenges of this paper and will be discussed in detail later on.

When necessary, we will employ the collapsed notation for HPLs and SVHPLs, which is related to the notation with explicit indices via

$$
\mathcal{L}_{a_{1}, a_{2}, \ldots} \leftrightarrow \mathcal{L}_{a_{1}-1}^{0 \ldots 0} 1 \underbrace{0 \ldots 0}_{a_{2}-1} 1 \ldots
$$

and equivalently for the HPLs. Note that in the collapsed notation commas are used to separate the indices, while in the explicit index notation no commas are present.

By either direct evaluation (for low orders) or by matching a suitable ansatz with the analytic properties and the class of integrals available, the functions $g_{n}^{(\ell)}$ have been determined completely up to five loops $[22,28,35]$ while results for LLA and NLLA are available for even higher loops. Note that direct evaluation of eq. (2.5) amounts to closing the integration contour at infinity in the positive or negative half-plane, depending on whether $|w| \lessgtr 1$, and summing up infinitely many residues, which for a given $n$ are located at $i \nu= \pm(|n| / 2+m)$, where $m$ is a positive integer.

A substantial improvement compared to earlier calculations made the determination of eq. (2.5) possible for $\mathrm{N}^{3}$ LLA and $\mathrm{N}^{4}$ LLA at the five-loop level: based on an observation made in ref. [36], the authors of ref. [35] recognized that the result in MRK can be obtained (up to subtleties to be discussed below) by calculating the result in a yet different kinematical limit on top of the MRK: the so-called double-scaling limit, $w^{*} \rightarrow 0$ with $w$ fixed. Once promoting the resulting harmonic polylogarithms to SVHPLs by simply replacing $H \rightarrow \mathcal{L}$, the six-point remainder function in MRK is nicely recovered from the double-scaling limit. The mechanism works upon assuming single-valuedness of the result and taking into account that the leading contribution to a SVHPL in the limit $w^{*} \rightarrow 0$, once we simply drop all logarithms $\log w^{*}$, is the corresponding harmonic polylogarithm (cf. eq. (2.15)) with the same index structure. 
Comparing with eq. (2.5), we see that we can implement this prescription by setting $w^{*}=1$ in the integrand, as well as only calculating the residue at $i \nu= \pm n / 2$. This automatically drops all $\operatorname{logarithms} \log w^{*}$ and by focusing on the residues at $i \nu= \pm n / 2$ we extract the $w^{*}$-independent part of the integral, which is the leading term in the limit $w^{*} \rightarrow 0$. The $w^{*}$-dependence is stored in the residues at $i \nu= \pm(n / 2+m)$, where $m$ is a positive integer, which we neglect. In this way, the integral will evaluate to HPLs, which we can then promote to their single-valued analogues to obtain the full result. In the following section we will thoroughly describe our method.

\section{Solving the integral}

Having the different approaches described above for evaluating eq. (2.5) at our disposal, it seems rather straightforward to evaluate the remainder function to high logarithmic and loop order. However, already at five loops the algebraic effort in matching a suitable ansatz to the sums resulting from the evaluation of the integral is considerable. Loop-orders higher than five are possible for low logarithmic orders, because those come with a rather small number of terms in the integrand. A solution for six loops including all logarithmic orders, however, seems out of reach. Removing this obstruction is precisely the main point of this article. There are two concepts our formalism is based upon, which will be described immediately.

Residues. The first ingredient is based on the observation made in refs. [35, 36] and described in the previous subsection: the full result of eq. (2.5) can be obtained by closing the integration contour below $w^{3}$ the real axis and considering the residue at $i \nu=n / 2$ with $n \geq 0$. Note that with our choice of writing the remainder function as in eq. (2.5), it is known that only half of the residue at $\nu=n=0$ has to be taken into account. This residue is closely tied to the Regge pole contribution. It should be noted that other approaches exist, in which this issue is circumvented by absorbing the Regge pole contribution into the integral, see ref. [39] for a detailed discussion on that point. Following ref. [22], we define a shorthand for the integrals in question:

$$
\mathcal{I}[\mathcal{F}(\nu, n)]=\frac{1}{\pi} \sum_{n=-\infty}^{\infty}(-1)^{n}\left(\frac{w}{w^{*}}\right)^{\frac{n}{2}} \int_{-\infty}^{\infty} \frac{\mathrm{d} \nu}{\nu^{2}+\frac{n^{2}}{4}}|w|^{2 i \nu} \mathcal{F}(\nu, n) .
$$

In order to further simplify the notation below we introduce:

$$
\mathcal{I} \bullet[\mathcal{F}(\nu, n)]=\left.\mathcal{I}[\mathcal{F}(\nu, n)]\right|_{i \nu=\frac{n}{2}, w^{*}=1} \quad \text { and } \quad \mathcal{I}_{0}[\mathcal{F}(\nu, n)]=\left.\frac{1}{2} \mathcal{I}[\mathcal{F}(\nu, n)]\right|_{\nu=n=0, w^{*}=1},
$$

where the above notation shall imply that in evaluating the integral only the contribution from residues noted are taken into account. Furthermore, the prescription in which only half of the residue at $\nu=n=0$ contributes to the integral is already included in the

\footnotetext{
${ }^{3}$ Closing the contour below corresponds to the case where $|w|<1$. We could equally well consider the case $|w|>1$ and close the contour above, which would lead to the same results.
} 
above definition. Naturally, the sum over $n$ will collapse in this case. Employing the above notation, the general technique used in this paper can be abbreviated by

$$
\mathcal{I}[\mathcal{F}(\nu, n)]=\left.\left(\mathcal{I}_{\bullet}[\mathcal{F}(\nu, n)]+\mathcal{I}_{0}[\mathcal{F}(\nu, n)]\right)\right|_{H \rightarrow \mathcal{L}} .
$$

Considering only poles in the lower half-plane or at $\nu=n=0$ is obviously simpler compared to the evaluation of the full integral: in particular we get rid of the dependence on the signature of $n$, as all $n$ 's in question are positive. Further simplifications in the classification of integrals below will occur upon splitting the lowest-order BFKL eigenvalue

$$
E^{(0)}=-\frac{1}{2}\left(\chi_{+}-\chi_{-}\right)+E_{\psi}
$$

into a part originating from the first term in eq. (2.11) and into a part $E_{\psi}$ containing the polygamma functions: while the former one does raise the order of the pole at $i \nu=n / 2$ in eq. (2.5), the contribution $E_{\psi}$ is free of poles at $i \nu=n / 2$. Note again that eq. (3.4) only holds because we are considering poles with $n \geq 0$ exclusively, as mentioned above.

Relations between residues. The second important ingredient to our formalism is to exploit relations between residues. In particular, we will derive a couple of rules which relate integrals among each other. In evaluating their residues, it will be very useful to rewrite particular values of (derivatives of) polygamma functions in terms of Euler $Z$-sums. Relating residues is the key to the efficiency of our method: instead of calculating numerous integrals by determining fudge coefficients of an ansatz, we calculate only very few integrals and express the residues of all other integrals in terms of those. The relations between the residues can be cast into a set of simple rules to be discussed below.

The formalism for the evaluation of eq. (2.5) can be summarized as follows:

- we expand the integrand in eq. (2.5) to the desired order in the coupling constant $a$ and use the relations (2.12) (and their higher-order generalizations), (2.13) and (3.4) to obtain several integrals of the general form

$$
\mathcal{I}\left[\chi_{+}^{n_{+}} \chi_{-}^{n_{-}}\left(\prod_{i} D_{\nu}^{a_{i}} E_{\psi}\right) E_{\psi}^{k}\right] .
$$

- We relate residues at $i \nu=n / 2$ of integrals of type (3.5) to residues of the basis integrals

$$
\mathcal{I}\left[\left(\prod_{i} D_{\nu}^{a_{i}} E_{\psi}\right) E_{\psi}^{k}\right] .
$$

- For the residues at $\nu=n=0$ we use a similar set of rules to again relate those to the residues at $\nu=n=0$ of the same basis integrals.

- Once all contributions to the integrals are calculated, we promote the harmonic polylogarithms to their single-valued cousins.

Using this approach and calculating a couple of basis integrals upfront allows a very fast determination of high orders of the remainder function. The results thus obtained match all known expressions. 


\subsection{Basic mechanism}

Let us get started by evaluating the simplest integral of type (3.5): $\mathcal{I}[1]$. One easily finds

$$
\mathcal{I}_{\bullet}[1]=\sum_{n=1}^{\infty} \frac{2(-w)^{n}}{n}=2 H_{1}(-w) \text {. }
$$

Similarly,

$$
\mathcal{I}_{0}[1]=\log w=H_{0}(w)
$$

which leads - after promoting the usual harmonic polylogarithms to their single-valued analogues $^{4}$ - to the known result

$$
\mathcal{I}[1]=\mathcal{L}_{0}(-w)+2 \mathcal{L}_{1}(-w)
$$

cf. eq. (2.15) with the identification $\left(z, z^{*}\right)=\left(-w,-w^{*}\right)$.

\subsection{Integrals of the form $\mathcal{I}\left[\chi_{+}^{n_{+}} \chi_{-}^{n_{-}}\right]$}

Integrals of the form $\mathcal{I}\left[\chi_{+}^{n_{+}} \chi_{-}^{n_{-}}\right]$are determined in such a way as to ensure single-valuedness of the resulting polylogarithm $\mathcal{L}_{s}(-w)$. Comparing eq. (2.19) with the following relation for the $w$-dependence of the integral $\mathcal{I}$ defined in eq. (3.1)

$$
-w \frac{\partial}{\partial w}\left(\chi_{-} w^{i \nu+\frac{n}{2}}\right)=w^{i \nu+\frac{n}{2}}
$$

it is natural to expect an insertion of $\chi_{-}$in the integrand to be related to prepending $\left(-x_{0}\right)$ to the result of the integral without the insertion. Accordingly, taking the derivative with respect to $w^{*}$, one finds

$$
-w^{*} \frac{\partial}{\partial w^{*}}\left(\chi_{+}\left(w^{*}\right)^{i \nu-\frac{n}{2}}\right)=\left(w^{*}\right)^{i \nu-\frac{n}{2}}
$$

which suggests that the insertion of $\chi_{+}$corresponds to appending a letter $\left(-y_{0}\right)=\left(-x_{0}\right)$ to the right. In fact, for the integrals considered in this paper we can explicitly show that this is correct on the level of the integral, i.e. we find

$$
\begin{aligned}
-w \partial_{w} \mathcal{I}\left[\chi_{+}^{n_{+}} \chi_{-}^{n_{-}+1}\left(\prod_{i} D_{\nu}^{a_{i}} E_{\psi}\right) E_{\psi}^{k}\right] & =\mathcal{I}\left[\chi_{+}^{n_{+}} \chi_{-}^{n_{-}}\left(\prod_{i} D_{\nu}^{a_{i}} E_{\psi}\right) E_{\psi}^{k}\right], \\
-w^{*} \partial_{w^{*}} \mathcal{I}\left[\chi_{+}^{n_{+}+1} \chi_{-}^{n_{-}}\left(\prod_{i} D_{\nu}^{a_{i}} E_{\psi}\right) E_{\psi}^{k}\right] & =\mathcal{I}\left[\chi_{+}^{n_{+}} \chi_{-}^{n_{-}}\left(\prod_{i} D_{\nu}^{a_{i}} E_{\psi}\right) E_{\psi}^{k}\right] .
\end{aligned}
$$

Since the proof of these formulas is not very insightful, the calculation can be found in appendix A. This leads us to the following rules for an insertion of $\chi_{ \pm}$in a general integrand:

\footnotetext{
${ }^{4}$ Note that the sign difference in the arguments of eqs. (3.7) and (3.8) is not a problem - in promoting $H_{0} \rightarrow \mathcal{L}_{0}$ we get another term $\log w^{*}$ which combines with the result of eq. (3.8) to $\log |w|^{2}$ for which the sign does not matter.
} 
- Insertion of $\chi_{-}$

Comparing eq. (3.12) with eq. (2.19) shows that the result of inserting $\chi_{-}$into an integral of type (3.5) is given by attaching $\left(-x_{0}\right)$ to the left of the result of the integral without insertion. The only subtlety arises through potential integration constants, which have to be $\zeta$-values to preserve uniform transcendentality. However, these constants can be easily determined from the residue at $\nu=n=0$ as will be discussed below.

- Insertion of $\chi_{+}$

Similarly, comparing eq. (3.13) with eq. (2.19) shows that inserting $\chi_{+}$in an integrand corresponds to attaching $\left(-x_{0}\right)$ to the right of the result of the integral without insertion. However, note from eq. (2.19) that the natural alphabet acting on the right-hand side of a SVHPL consists of the letters $y_{0}$ and $y_{1}$. Because of this on top of the simple prescription of appending $\left(-x_{0}\right)$ - we find additional terms containing $\zeta$-values when inserting a factor $\chi_{+}$in the integrand. These additional terms are exactly those needed to preserve eq. (3.13). Let us clarify this point with an example: assume we have an integral which evaluates to $\mathcal{L}_{101}$. If we insert a factor $\chi_{+}$in the integrand we naïvely expect to find the result $-\mathcal{L}_{1010}$. However, this result violates relation (3.13) since

$$
-w^{*} \partial_{w^{*}}\left(-\mathcal{L}_{1010}\right)=\mathcal{L}_{101}+4 \zeta_{3} \frac{w^{*}}{1+w^{*}},
$$

as can be easily checked from eq. (2.16). To preserve relation (3.13) the true integral after the insertion of $\chi_{+}$will therefore read $-\mathcal{L}_{1010}-4 \zeta_{3} \mathcal{L}_{1}$. In summary, when inserting factors of $\chi_{+}$in an integrand, on top of simply appending factors of $\left(-x_{0}\right)$ to the right, we will find additional contributions containing $\zeta$-values which arise exactly to preserve the differential equations (3.13).

In section 3.4 we are going to show how to get the complications of the $\zeta$-values under control by using recursion relations which relate residues of different integrals. It should be noted, however, that the complications beyond simply attaching factors of $\left(-x_{0}\right)$ for insertions of $\chi_{ \pm}$always come with $\zeta$-values, which in turn means that the $\zeta$-free parts are fully understood from eqs. (3.12) and (3.13).

Before closing this section, let us write down specific examples of the formulæ (3.12), (3.13) in the simplest case

$$
\mathcal{I}\left[\chi_{+}\right]=-\mathcal{I}[1] x_{0} \quad \text { and } \quad \mathcal{I}\left[\chi_{-}\right]=-x_{0} \mathcal{I}[1]
$$

The notation in the equation above means that letters $x_{0}$ are concatenated to each of the words in $\mathcal{I}[1]$, using the identification eq. (2.14). For the first and second part of eq. (3.15) we need to append $\left(-x_{0}\right)$ to the right and to the left, respectively, in order to find from eq. (3.9)

$$
\mathcal{I}\left[\chi_{+}\right]=-\mathcal{L}_{00}(-w)-2 \mathcal{L}_{10}(-w) \quad \text { and } \quad \mathcal{I}\left[\chi_{-}\right]=-\mathcal{L}_{00}(-w)-2 \mathcal{L}_{01}(-w)
$$


Equation (3.15) can be easily generalized to

$$
\mathcal{I}\left[\chi_{+}^{n_{+}} \chi_{-}^{n_{-}}\right]=(-1)^{n_{+}+n_{-}} x_{0}^{n_{-}} \mathcal{I}[1] x_{0}^{n_{+}} .
$$

One might wonder, why for higher orders of the insertion $\chi_{+}$there is no appearance of any $\zeta$ 's which can be attributed to the alphabet $y_{0}, y_{1}$. The naïve reason is that the initial integral $\mathcal{I}[1]$ contains exactly one letter $x_{1}$. As powers of $\chi_{+}$and $\chi_{-}$lead to appending letters $\left(-x_{0}\right)$ only, there will never be a polylogarithm corresponding to a word with more than one letter $x_{1}$. Comparing with (also higher orders of) eq. (2.20), one recognizes that any $\zeta$-contribution comes with words containing at least two letters $x_{1}$. As those can never be created by appending $x_{0}$ to $\mathcal{I}[1]$, there will be no $\zeta$-corrections to eq. (3.17).

\subsection{Basis integrals}

Before proceeding to rules for general integrals of the form eq. (3.5), let us consider what we call basis integrals, that is those without any insertions of $\chi_{+}$and $\chi_{-}$:

$$
\mathcal{I}\left[\left(\prod_{i} D_{\nu}^{a_{i}} E_{\psi}\right) E_{\psi}^{k}\right]
$$

The residue of those integrals at $i \nu=n / 2$ is most efficiently calculated employing Euler $Z$-sums, a technology which has already been employed in the context of the Wilson loop OPE in refs. [35, 40]. The mechanism works by recognizing that neither $E_{\psi}$ nor its derivatives have any poles at $i \nu=n / 2$. Therefore any pole at $i \nu=n / 2$ with $n>0$ in the basis integrals will be of first order (cf. eq. (3.1)) and the integral can thus be easily calculated. Evaluating derivatives on $E_{\psi}$ one finds:

$$
\begin{aligned}
D_{\nu}^{k} E_{\psi} & =\psi^{(k)}(1+i \nu+n / 2)+(-1)^{k} \psi^{(k)}(1-i \nu+n / 2) \\
\left.D_{\nu}^{k} E_{\psi}\right|_{i \nu=n / 2} & =\psi^{(k)}(1+n)+(-1)^{k} \psi^{(k)}(1) .
\end{aligned}
$$

Employing

$$
\psi^{(m)}(n)=(-1)^{m+1} m !\left(\zeta(m+1)-Z_{m+1}(n-1)\right),
$$

where by definition

$$
Z_{m}(n):=\sum_{\ell=1}^{n} \frac{1}{\ell^{m}}, \quad \text { and } \quad Z_{m_{1}, \ldots, m_{k}}(n):=\sum_{\ell=1}^{n} \frac{1}{\ell^{m_{1}}} Z_{m_{2}, \ldots, m_{k}}(\ell-1),
$$

and generalizing to several powers of the derivative $D_{\nu}$, yields

$$
\mathcal{I}_{\bullet}\left[\left(D_{\nu}^{k} E_{\psi}\right)^{q}\right]=\sum_{n=1}^{\infty}(-1)^{k} k ! \frac{2(-w)^{n}}{n}\left(Z_{k+1}(n)-\left(1+(-1)^{k}\right) \zeta_{k+1}\right)^{q}
$$


For several powers of the function $E_{\psi}$ the residue reads

$$
\mathcal{I}_{\bullet}\left[E_{\psi}^{q}\right]=\sum_{n=1}^{\infty} \frac{2(-w)^{n}}{n} Z_{1}(n)^{q} .
$$

The resulting $Z$-sums can now be converted into polylogarithms: this is done with ease employing the identity (for this and the following identities see [41] $)^{5}$

$$
H_{a s}(w)=\sum_{k=1}^{\infty} \frac{w^{k}}{k^{a}} Z_{s}(\ell-1)
$$

and - in order to shift the argument of the $Z$-sum to have the appropriate form -

$$
Z_{m_{1}, \ldots, m_{k}}(n+c-1)=Z_{m_{1}, \ldots, m_{k}}(n-1)+\sum_{j=0}^{c-1} \frac{1}{(n+j)^{m_{1}}} Z_{m_{2}, \ldots, m_{k}}(n+j-1) .
$$

The identity eq. (3.24) above, however, can be applied to single $Z$-sums exclusively. Therefore products of $Z$-sums of the same argument have to be converted into single $Z$-sums, which can be done by recursively using the stuffle relation

$$
\begin{aligned}
Z_{m_{1}, \ldots, m_{p}}(n) Z_{m_{1}^{\prime}, \ldots, m_{q}^{\prime}}(n)= & \sum_{i_{1}=1}^{n} \frac{1}{i_{1}^{m_{1}}} Z_{m_{2}, \ldots, m_{p}}\left(i_{1}-1\right) Z_{m_{1}^{\prime}, \ldots, m_{q}^{\prime}}\left(i_{1}-1\right) \\
& +\sum_{i_{2}=1}^{n} \frac{1}{i_{2}^{m_{1}^{\prime}}} Z_{m_{1}, \ldots, m_{p}}\left(i_{2}-1\right) Z_{m_{2}^{\prime}, \ldots, m_{q}^{\prime}}\left(i_{2}-1\right) \\
& +\sum_{i=1}^{n} \frac{1}{i^{m_{1}+m_{1}^{\prime}}} Z_{m_{2}, \ldots, m_{p}}(i-1) Z_{m_{2}^{\prime}, \ldots, m_{q}^{\prime}}(i-1) .
\end{aligned}
$$

In order to evaluate the full integral we also need to consider the residues at the double pole $\nu=n=0$. The function $E_{\psi}$ vanishes at $\nu=n=0$, which lets the residue vanish as soon as any power greater than 1 of $E_{\psi}$ is present in any basis integral. As soon as there are, however, derivatives of $E_{\psi}$ only, there will be a contribution. The general case can be calculated by simply expanding the relation

$$
\mathcal{I}_{0}\left[\left(\prod_{i} D_{\nu}^{a_{i}} E_{\psi}\right) E_{\psi}^{k}\right]=\left.D_{\nu}\left(w^{i \nu}\left(\prod_{i} D_{\nu}^{a_{i}} E_{\psi}\right) E_{\psi}^{k}\right)\right|_{\nu=0}
$$

from which we find the special cases

$$
\begin{aligned}
\mathcal{I}_{0}\left[E_{\psi}^{q}\right] & \left.\sim E_{\psi}^{q-1}\right|_{\nu=0}=0, \quad \text { for } q>1 \\
\mathcal{I}_{0}\left[D_{\nu}^{k} E_{\psi}\right] & =\left\{\begin{array}{lll}
-2(k+1) ! \zeta_{k+2} & k & \text { odd } \\
-2 k ! H_{0}(w) \zeta_{k+1} & k & \text { even }
\end{array}\right.
\end{aligned}
$$

for $k>0$. This completes our discussion of the basis integrals and we now move on to explain the calculation of a general integral.

\footnotetext{
${ }^{5}$ Note that eq. (3.24) only holds if the index does not contain trailing 0's in which case the harmonic polylogarithm contains logarithmic terms. However, in the integrals we consider this is never the case.
} 


\subsection{General integral}

We are now ready to tackle the case of a general integrand. The analysis will be split into a part in which we describe the recursive structure of the residues at $i \nu=n / 2$, followed by a second part, in which we carry out the analysis of the residue at $\nu=n=0$.

Residue at $\boldsymbol{i} \boldsymbol{\nu}=\boldsymbol{n} / \mathbf{2}$. From the above analysis it became clear that adjoining any power of $\chi_{-}$to an integrand $\mathcal{F}$ will result in prepending the appropriate number of letters $x_{0}$ to the result $\mathcal{I}[\mathcal{F}]$. The only subtlety is the potential appearance of $\zeta$-values as integration constants, which, however, only come from the residue at $\nu=n=0$ and will be discussed later. We can therefore exclude powers of $\chi_{-}$in the analysis below and focus on insertions of $\chi_{+}$.

In order to derive the relation between different residues at $i \nu=n / 2$, let us get started with the most general integrand not containing $\chi_{-}$and calculate its residue:

$$
\begin{aligned}
\mathcal{I}_{\bullet} & {\left[\chi_{+}^{k+1}\left(\prod_{i} D^{a_{i}} E_{\psi}\right) E_{\psi}^{q}\right] } \\
& =\left.(-2 i) \sum_{n=1}^{\infty}(-1)^{n} \frac{(-1)^{k+1}}{(k+1) !} D_{\nu}^{k+1}\left(\frac{w^{i \nu+n / 2}}{\nu-\frac{i n}{2}}\left(\prod_{i} D^{a_{i}} E_{\psi}\right) E_{\psi}^{q}\right)\right|_{\nu=-\frac{i n}{2}}
\end{aligned}
$$

Aiming for a recursion, we will perform just one of the derivatives $D_{\nu}$, yielding

$$
\begin{gathered}
=(-2 i) \sum_{n}(-1)^{n} \frac{(-1)^{k+1}}{(k+1) k !} D_{\nu}^{k}\left(\log w \frac{w^{i \nu+n / 2}}{\nu-\frac{i n}{2}}\left(\prod_{i} D_{\nu}^{a_{i}} E_{\psi}\right) E_{\psi}^{q}+i \frac{w^{i \nu+n / 2}}{\left(\nu-\frac{i n}{2}\right)^{2}}\left(\prod_{i} D_{\nu}^{a_{i}} E_{\psi}\right) E_{\psi}^{q}\right. \\
\left.+\frac{w^{i \nu+n / 2}}{\nu-\frac{i n}{2}} D_{\nu}\left(\left(\prod_{i} D_{\nu}^{a_{i}} E_{\psi}\right) E_{\psi}^{q}\right)\right)\left.\right|_{\nu=-\frac{i n}{2}} .
\end{gathered}
$$

The above expression can be rewritten as a sum of integrals evaluated at $i \nu=n / 2$

$$
\begin{gathered}
=\frac{1}{(k+1)}\left(-H_{0}(w) \mathcal{I}_{\bullet}\left[\chi_{+}^{k}\left(\prod_{i} D_{\nu}^{a_{i}} E_{\psi}\right) E_{\psi}^{q}\right]-\mathcal{I}_{\bullet}\left[\chi_{+}^{k} \chi_{-}\left(\prod_{i} D_{\nu}^{a_{i}} E_{\psi}\right) E_{\psi}^{q}\right]\right. \\
\left.-\mathcal{I}_{\bullet}\left[\chi_{+}^{k} D_{\nu}\left(\left(\prod_{i} D_{\nu}^{a_{i}} E_{\psi}\right) E_{\psi}^{q}\right)\right]\right) .
\end{gathered}
$$

The integrands on the right-hand side of the above equation have less powers of $\chi_{+}$compared to the integrand we started with in eq. (3.29). Therefore we can successively get rid of all powers of $\chi_{+}$by trading them for easier integrals. At the end of the recursion we are left with just the basis integrals discussed in subsection 3.3 above and insertions of $\chi_{-}$, which we understand.

Residue at $\boldsymbol{\nu}=\boldsymbol{n}=\mathbf{0}$. For the second residue contributing to the calculation of the remainder function, the analysis is slightly simpler, but follows a similar pattern. Again, we start from the most general integral, however, this time we have to consider powers of $\chi_{-}$as well, as those contribute in the same way as $\chi_{+}$: for $n=0$ both of them reduce to 
$i / \nu$ (cf. eqs. (2.13) and (2.9)). Starting from an integral with $k$ powers of either $\chi_{+}$or $\chi_{-}$, we find

$$
\mathcal{I}_{0}\left[\left(\frac{i}{\nu}\right)^{k}\left(\prod_{i} D^{a_{i}} E_{\psi}\right) E_{\psi}^{q}\right]=\left.(-1)^{k} \frac{D_{\nu}^{k+1}}{(k+1) !}\left(w^{i \nu}\left(\prod_{i} D^{a_{i}} E_{\psi}\right) E_{\psi}^{q}\right)\right|_{\nu=0} .
$$

Performing again just one of the derivatives $D_{\nu}$, yields

$$
=\left.(-1)^{k} \frac{D_{\nu}^{k}}{(k+1) !}\left(w^{i \nu} \log w\left(\prod_{i} D^{a_{i}} E_{\psi}\right) E_{\psi}^{q}+w^{i \nu} D_{\nu}\left(\left(\prod_{i} D_{\nu}^{a_{i}} E_{\psi}\right) E_{\psi}^{q}\right)\right)\right|_{\nu=0}
$$

which is again the sum of several different residues:

$$
=\frac{1}{(k+1)}\left(-H_{0}(w) \mathcal{I}_{0}\left[\left(\frac{i}{\nu}\right)^{k-1}\left(\prod_{i} D_{\nu}^{a_{i}} E_{\psi}\right) E_{\psi}^{q}\right]-\mathcal{I}_{0}\left[\left(\frac{i}{\nu}\right)^{k-1} D_{\nu}\left(\left(\prod_{i} D_{\nu}^{a_{i}} E_{\psi}\right) E_{\psi}^{q}\right)\right]\right) .
$$

Similar to the recursive formalism used above for the contribution from $i \nu=n / 2$, one can thus recursively trace back any situation to the residues at $\nu=n=0$ of the basis integrals.

\subsection{Example: $\mathcal{I}\left[\left(D_{\nu}^{2} E\right) E\right]$}

In order to illustrate the above method, let us consider a simple but nontrivial example. The integral $\mathcal{I}\left[\left(D_{\nu}^{2} E\right) E\right]$, which appears at four loops in NLLA, can be expanded as

$$
\begin{aligned}
\mathcal{I}\left[\left(D_{\nu}^{2} E\right) E\right]= & \mathcal{I}\left[\frac{\chi_{-}^{4}}{2}-\frac{\chi_{-}^{3} \chi_{+}}{2}-\frac{\chi_{-} \chi_{+}^{3}}{2}+\frac{\chi_{+}^{4}}{2}+\chi_{-}^{3} E_{\psi}-\chi_{+}^{3} E_{\psi}\right. \\
& \left.+\frac{\chi_{-} D_{\nu}^{2} E_{\psi}}{2}-\frac{\chi_{+} D_{\nu}^{2} E_{\psi}}{2}+E_{\psi} D_{\nu}^{2} E_{\psi}\right]
\end{aligned}
$$

The integrals, which contain powers of $\chi_{+}$and $\chi_{-}$only, can be trivially solved using eq. (3.17),

$$
\mathcal{I}\left[\frac{\chi_{-}^{4}}{2}-\frac{\chi_{-}^{3} \chi_{+}}{2}-\frac{\chi_{-} \chi_{+}^{3}}{2}+\frac{\chi_{+}^{4}}{2}\right]=\mathcal{L}_{00001}-\mathcal{L}_{00010}-\mathcal{L}_{01000}+\mathcal{L}_{00001}
$$

where we suppressed the arguments $w$ of the SVHPLs. Using the direct methods described in subsection 3.3 in order to derive the basis integrals

$$
\begin{aligned}
\mathcal{I}\left[E_{\psi}\right] & =\left.\left(\mathcal{I}_{\bullet}\left[E_{\psi}\right]+\mathcal{I}_{0}\left[E_{\psi}\right]\right)\right|_{H \rightarrow \mathcal{L}}=2\left(\mathcal{L}_{01}+\mathcal{L}_{11}\right), \\
\mathcal{I}\left[D_{\nu}^{2} E_{\psi}\right] & =\left.\left(\mathcal{I}_{\bullet}\left[D_{\nu}^{2} E_{\psi}\right]+\mathcal{I}_{0}\left[D_{\nu}^{2} E_{\psi}\right]\right)\right|_{H \rightarrow \mathcal{L}}=4\left(\mathcal{L}_{0001}+\mathcal{L}_{1001}-2 \zeta_{3} \mathcal{L}_{1}\right)-4 \zeta_{3} \mathcal{L}_{0}, \\
\mathcal{I}\left[E_{\psi} D_{\nu}^{2} E_{\psi}\right] & =4\left(\mathcal{L}_{00001}+\mathcal{L}_{00011}+\mathcal{L}_{01001}+\mathcal{L}_{10001}+\mathcal{L}_{10011}+\mathcal{L}_{11001}-2 \zeta_{3}\left(\mathcal{L}_{01}+\mathcal{L}_{11}\right)\right),
\end{aligned}
$$


one can now tackle the other integrals. By just attaching the appropriate number of letters $\left(-x_{0}\right)$ from the left matching the power of $\chi_{-}$, one finds immediately

$$
\begin{aligned}
\mathcal{I}\left[\chi_{-}^{3} E_{\psi}\right] & =\left(-x_{0}\right)^{3}\left(\left.\mathcal{I}_{\bullet}\left[E_{\psi}\right]\right|_{H \rightarrow \mathcal{L}}\right)+\left.\mathcal{I}_{0}\left[\chi_{-}^{3} E_{\psi}\right]\right|_{H \rightarrow \mathcal{L}} \\
& =-2\left(\mathcal{L}_{00001}+\mathcal{L}_{00011}\right)+2 \mathcal{L}_{00} \zeta_{3}+2 \zeta_{5}, \\
\mathcal{I}\left[\chi_{-} D_{\nu}^{2} E_{\psi}\right] & =-x_{0}\left(\left.\mathcal{I}_{\bullet}\left[D_{\nu}^{2} E_{\psi}\right]\right|_{H \rightarrow \mathcal{L}}\right)+\left.\mathcal{I}_{0}\left[\chi_{-} D_{\nu}^{2} E_{\psi}\right]\right|_{H \rightarrow \mathcal{L}} \\
& =-4\left(\mathcal{L}_{00001}+\mathcal{L}_{01001}-2 \zeta_{3} \mathcal{L}_{01}\right)+4\left(\zeta_{3} \mathcal{L}_{00}+6 \zeta_{5}\right) \\
& =-4\left(\mathcal{L}_{00001}+\mathcal{L}_{01001}-\zeta_{3}\left(\mathcal{L}_{00}+2 \mathcal{L}_{01}\right)-6 \zeta_{5}\right) .
\end{aligned}
$$

Let us now evaluate the integral $\mathcal{I}\left[\chi_{+}^{3} E_{\psi}\right]$. In order to get started, let us consider the residue at $i \nu=n / 2$, which we will rewrite using eq. (3.31):

$$
\mathcal{I}_{\bullet}\left[\chi_{+}^{3} E_{\psi}\right]=\frac{1}{3}\left(-H_{0}(w) \mathcal{I}_{\bullet}\left[\chi_{+}^{2} E_{\psi}\right]-\mathcal{I}_{\bullet}\left[\chi_{+}^{2} \chi_{-} E_{\psi}\right]-\mathcal{I}_{\bullet}\left[\chi_{+}^{2} D_{\nu} E_{\psi}\right]\right)
$$

Naturally, one would now reduce $\mathcal{I}_{\bullet}\left[\chi_{+}^{2} E_{\psi}\right]$ in the same manner and finally continue with the integral $\mathcal{I}_{\bullet}\left[\chi_{+} E_{\psi}\right]$. After evaluating everything, one finds

$$
\begin{aligned}
& \mathcal{I}_{\bullet}\left[\chi_{+}^{3} E_{\psi}\right]=-2\left(H_{01000}+H_{11000}\right)+4 \zeta_{3} H_{10}, \\
& \mathcal{I}_{\bullet}\left[\chi_{+}^{2} E_{\psi}\right]=2\left(H_{0100}+H_{1100}\right)-4 \zeta_{3} H_{1}, \\
& \mathcal{I}_{\bullet}\left[\chi_{+} E_{\psi}\right]=-2\left(H_{010}+H_{110}\right)
\end{aligned}
$$

What remains to be done is to calculate the contributions from the residue at $\nu=n=0$ for the above integrals. This can be done in (almost) complete analogy to the considerations above by using eq. (3.34). One finds:

$$
\begin{aligned}
& \mathcal{I}_{0}\left[\chi_{+}^{3} E_{\psi}\right]=2 \zeta_{3} H_{00}+2 \zeta_{5}, \\
& \mathcal{I}_{0}\left[\chi_{+}^{2} E_{\psi}\right]=-2 \zeta_{3} H_{0}, \\
& \mathcal{I}_{0}\left[\chi_{+} E_{\psi}\right]=2 \zeta_{3} .
\end{aligned}
$$

Adding up all contributions and using the replacement rule (3.3), one finally finds:

$$
\begin{aligned}
\mathcal{I}\left[\left(D_{\nu}^{2} E\right) E\right]= & \mathcal{L}_{00001}+\mathcal{L}_{00010}+2 \mathcal{L}_{00011}+\mathcal{L}_{01000}+2 \mathcal{L}_{01001} \\
& +\mathcal{L}_{10000}+4 \mathcal{L}_{10001}+2 \mathcal{L}_{10010}+4 \mathcal{L}_{10011}+2 \mathcal{L}_{11000} \\
& +4 \mathcal{L}_{11001}-4 \zeta_{3}\left(\mathcal{L}_{01}+2 \mathcal{L}_{10}+2 \mathcal{L}_{11}\right)
\end{aligned}
$$

As should be clear from our example, these calculations will become tedious for high weights if carried out by hand, but are very simple to implement on a computer. In particular, given the recursive structure there are very few integrals to be determined at all. 


\section{NMHV}

While the discussion in the previous sections was tailored to obtaining the six-point remainder function $R_{6}^{\mathrm{MHV}}$, we would like to apply our method to the NMHV sector. Following the setup described in ref. [37], we flip the helicity of the external gluon 4 compared to the MHV-situation in order to obtain the helicity configuration $(++--+-) .{ }^{6}$ Naturally, the kinematical setup remains the same as described in eqs. (2.2) and (2.3). Below we are going to relate the integrals appearing in the NMHV sector to those which have already been solved for the MHV sector above. This idea has already been proven useful in many situations $[22,27,37]$ and has been discussed therein thoroughly. Therefore, we sketch the formalism only briefly and point out where particular adjustments to our setup are necessary. The remainder function $R_{6}^{\mathrm{NMHV}}$ is defined via

$$
R_{6}^{\mathrm{NMHV}}=\frac{A_{\mathrm{NMHV}}}{A_{\mathrm{BDS}}}=\mathcal{P}_{\mathrm{NMHV}} \times e^{R_{6}^{\mathrm{MHV}}}
$$

and can be determined by evaluating the following integral [37]:

$$
\begin{aligned}
\mathcal{P}_{\mathrm{NMHV}} \times\left. e^{R+i \pi \delta}\right|_{\mathrm{MRK}}= & \cos \pi \omega_{a b}-i \frac{a}{2} \sum_{n=-\infty}^{\infty}(-1)^{n}\left(\frac{w}{w^{*}}\right)^{\frac{n}{2}} \int_{-\infty}^{\infty} \frac{\mathrm{d} \nu}{\left(i \nu+\frac{n}{2}\right)^{2}}|w|^{2 i \nu} \Phi_{\mathrm{reg}}^{\mathrm{NMHV}}(\nu, n) \\
& \times \exp \left[-\omega(\nu, n)\left(\log \left(1-u_{1}\right)+i \pi+\frac{1}{2} \log \frac{|w|^{2}}{|1+w|^{4}}\right)\right] .
\end{aligned}
$$

The above formula differs from eq. (2.5) in two respects: the factor determining the residue in each integral is altered via

$$
\frac{1}{\nu^{2}+\frac{n^{2}}{4}}=-\chi_{+} \chi_{-} \rightarrow-\frac{1}{\left(i \nu+\frac{n}{2}\right)^{2}}=-\chi_{-}^{2}
$$

and the MHV impact factor is replaced by $\Phi_{\text {reg }}^{\mathrm{NMHV}}$, the impact factor for NMHV. It can be obtained at any loop order by supplementing $\Phi_{\text {reg }}^{\mathrm{MHV}}$ with a suitable correction factor, which, in turn, can be inferred and derived from integrability [29, 37].

Having all the ingredients for the integrand available, we are left with evaluating the integral. Fortunately one does not explicitly need to solve this particular integral: defining

$$
\mathcal{I}^{\mathrm{NMHV}}[\mathcal{F}(\nu, n)]=\frac{1}{\pi} \sum_{n=-\infty}^{\infty}(-1)^{n}\left(\frac{w}{w^{*}}\right)^{\frac{n}{2}} \int_{-\infty}^{\infty} \frac{\mathrm{d} \nu}{\left(i \nu+\frac{n}{2}\right)^{2}}|w|^{2 i \nu} \mathcal{F}(\nu, n),
$$

one easily finds from eqs. (3.10) and (3.11) that

$$
w \frac{\partial}{\partial w} \mathcal{I}^{\mathrm{NMHV}}[\mathcal{F}(\nu, n)]=-w^{*} \frac{\partial}{\partial w^{*}} \mathcal{I}^{\mathrm{MHV}}[\mathcal{F}(\nu, n)],
$$

where $\mathcal{I}^{\mathrm{MHV}}$ refers to the integral defined in eq. (3.1). Derivatives with respect to the variable $w^{*}$, however, can be easily performed using eq. (2.19) after taking the identification $\left(z, z^{*}\right)=\left(-w,-w^{*}\right)$ into account.

\footnotetext{
${ }^{6}$ Note that in the high-energy limit, only the helicities of the external gluons 4 and 5 can be flipped, since helicity is conserved along the high-energy lines.
} 
The ratio function $\mathcal{P}_{\mathrm{NMHV}}$ can be expanded in the loop order as

$$
\begin{aligned}
\left.\mathcal{P}_{\mathrm{NMHV}}\right|_{\mathrm{MRK}}= & 1+\sum_{\ell=2}^{\infty} \sum_{n=0}^{\ell-1} a^{\ell} \log ^{n}\left(1-u_{1}\right)\left\{\frac{1}{1+w^{*}}\left[p_{n}^{(\ell)}\left(w, w^{*}\right)+2 \pi i q_{n}^{(\ell)}\left(w, w^{*}\right)\right]\right. \\
& \left.+\left.\frac{w^{*}}{1+w^{*}}\left[p_{n}^{(\ell)}\left(w, w^{*}\right)+2 \pi i q_{n}^{(\ell)}\left(w, w^{*}\right)\right]\right|_{\left(w, w^{*}\right) \rightarrow\left(1 / w, 1 / w^{*}\right)}\right\} .
\end{aligned}
$$

Starting from eq. (4.2), one will have to solve the integral, plug in the appropriate functions $g_{n}^{(\ell)}$ and $h_{n}^{(\ell)}$ for $R_{6}^{\mathrm{MHV}}$ and bring the result into the form (4.6) to determine the functions $p_{n}^{(\ell)}$ and $q_{n}^{(\ell)}$. Following this method, we can confirm all previous results in refs. [27, 37] and [42]. Higher orders can be obtained with the same ease and comparable computational effort as in the MHV case.

\section{Applications}

Using our algorithm as described above, we can straightforwardly produce new data for a high number of loops. As specific applications of our technique, we therefore present plots of the imaginary parts $g_{\ell-4}^{(\ell)}$ at $\mathrm{N}^{3}$ LLA up to ten loops and $g_{\ell-5}^{(\ell)}$ up to nine loops, as well as new expressions for the remainder function in the collinear-Regge limit.

\section{$5.1 \quad \mathrm{~N}^{3} \mathrm{LLA} / \mathrm{N}^{4} \mathrm{LLA}$}

As the simplest application of our algorithm, we investigate the behavior of the previouslyunknown functions $g_{\ell-4}^{(\ell)}, g_{\ell-5}^{(\ell)}$ beyond five loops. In figures 1 and 2 we plot these functions along the line $w=w^{*}$ up to ten loops for $\mathrm{N}^{3} \mathrm{LLA}$ and nine loops for $\mathrm{N}^{4} \mathrm{LLA}$. To produce these plots, we have used GiNaC [43] for the numerical evaluation of the HPLs.

Let us briefly comment on the performance of our algorithm. Using the most straightforward implementation of our algorithm in Mathematica on a standard laptop, we obtain the expressions up to ten loops for LLA and NLLA in about one minute each and for $\mathrm{N}^{3} \mathrm{LLA}$ in about ten minutes. We furthermore determined the full remainder function in the high-energy limit up to seven loops by calculating $g_{k}^{(7)}$ for $k=0, \ldots, 6$, which took of the order of 100 minutes, due to the high logarithmic degree for $k=0$. It should be possible to reduce these numbers a lot by a more sophisticated implementation. However, usually we do not stop at the expression in terms of SVHPLs, but rather want to compute series expansions or generate data points such as those shown in figures 1, 2. To do so, we need to know the expressions of the SVHPLs in terms of HPLs up to high weights, which, in turn, requires the solution of the single-valuedness condition in ref. [34] for high weights. Both solving the single-valuedness condition as well as series expansions or numerical evaluations of the HPLs become time-consuming for high weights. Also taking into account that the number of terms appearing in the $g_{n}^{(\ell)}$ grows with the logarithmic degree, we think that it should be possible to obtain results a couple of orders beyond the results presented here, but not much further. 


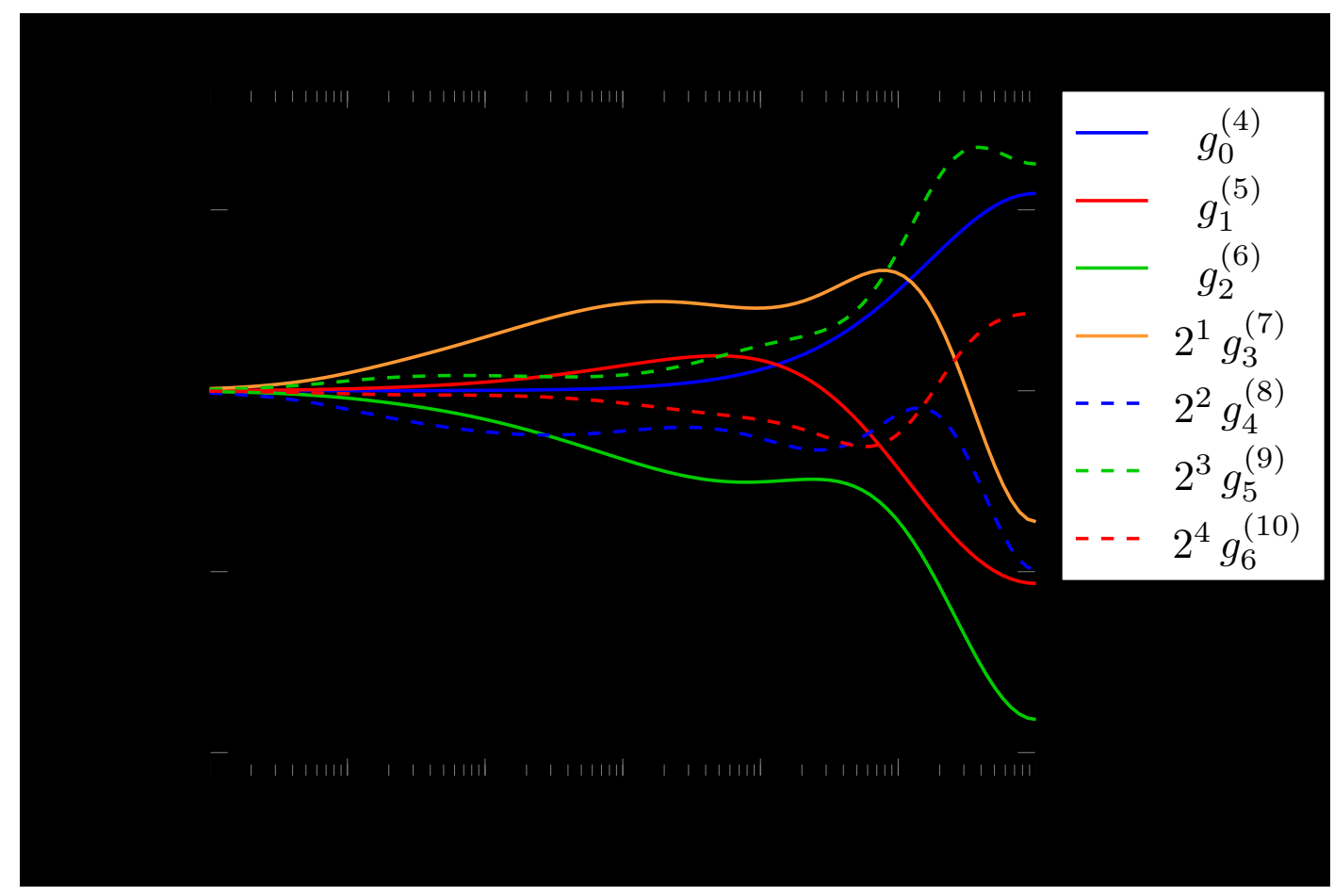

Figure 1. Behavior of the $\mathrm{N}^{3}$ LLA-functions $g_{\ell-4}^{(\ell)}$ along the line $w=w^{*}$. Note that some of the functions have been rescaled for convenience.

\subsection{Collinear-Regge limit}

As described in eq. (2.3) the remaining kinematical freedom in the multi-Regge limit is described by the complex variable $w$. We therefore have the freedom of taking another limit on top of the multi-Regge limit,

$$
|w| \rightarrow 0
$$

which describes the collinear-Regge regime. In this limit, we in principle have two different kinds of large logarithms, namely $\log \left(1-u_{1}\right)$ and $\log |w|$. However, the remainder function organizes itself effectively as a function of a single variable,

$$
x:=a \log \left(1-u_{1}\right) \log |w| .
$$

Indeed, the leading contributions, i.e. those with the maximal powers of $\log |w|$ at a given loop order, in the LLA collinear-Regge remainder function can be explicitly resummed in a very simple form

$$
\left.R_{6}^{\mathrm{MHV}}\right|_{\text {LLA,coll. }}=i \pi a\left(w+w^{*}\right)\left(1-I_{0}(2 \sqrt{x})\right),
$$

where $I_{0}(x)$ is a modified Bessel function, cf. [44]. These leading contributions are also referred to as the double-leading-logarithmic approximation (DLLA), with an obvious generalization beyond LLA. Note that the prefactor $\left(w+w^{*}\right)$ in eq. (5.3) is necessary to ensure that the remainder function vanishes in the collinear limit. A closed formula for all subleading contributions was presented in ref. [23]. 


\section{$\mathrm{N}^{4} \mathrm{LLA}$}

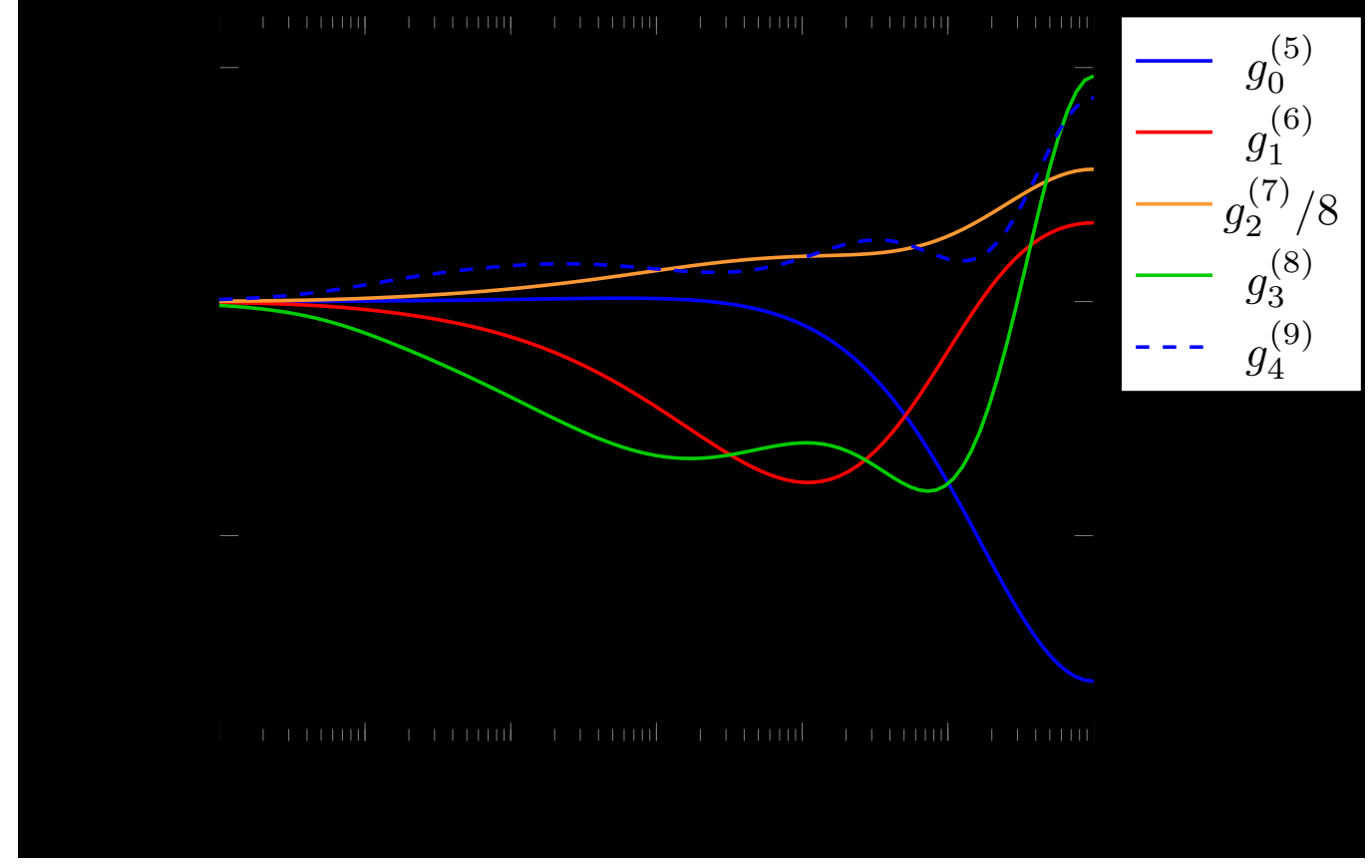

Figure 2. Behavior of the $\mathrm{N}^{4}$ LLA-functions $g_{\ell-5}^{(\ell)}$ along the line $w=w^{*}$. Note that some of the functions have been rescaled for convenience.

Using our algorithm, we can generate data up to a high number of loops. Finding the leading contributions in the collinear-Regge limit is then simply a matter of expanding the appearing SVHPLs in the limit $|w| \rightarrow 0$. This allows us to write down formulas for the leading contributions to the imaginary part of the remainder function in the collinear-Regge limit for $\mathrm{N}^{(1,2,3)} \mathrm{LLA}$ :

$$
\begin{aligned}
\left.\operatorname{Im} R_{6}^{\mathrm{MHV}}\right|_{\mathrm{DNLLA}}= & 2 \pi a^{2} \log |w|\left(w+w^{*}\right)\left(-\frac{1}{2} I_{0}(2 \sqrt{x})-\frac{1}{2} \partial_{x} I_{0}(2 \sqrt{x})\right) \\
\left.\operatorname{Im} R_{6}^{\mathrm{MHV}}\right|_{\mathrm{DN} 2 \mathrm{LLA}}= & 2 \pi a^{3} \log ^{2}|w|\left(w+w^{*}\right)\left[\left(-\frac{1}{4} I_{0}(2 \sqrt{x})-\partial_{x} I_{0}(2 \sqrt{x})-\frac{1}{2} \partial_{x}^{2} I_{0}(2 \sqrt{x})\right)\right. \\
& \left.+\zeta_{2}\left(\frac{3}{2} I_{0}(2 \sqrt{x})-2 \partial_{x} I_{0}(2 \sqrt{x})+\frac{3}{2} \partial_{x}^{2} I_{0}(2 \sqrt{x})\right)\right] \\
\left.\operatorname{Im} R_{6}^{\mathrm{MHV}}\right|_{\mathrm{DN}{ }^{3} \mathrm{LLA}}= & 2 \pi a^{4} \log ^{3}|w|\left(w+w^{*}\right)\left[\left(-\frac{1}{12} I_{0}(2 \sqrt{x})-\frac{3}{4} \partial_{x} I_{0}(2 \sqrt{x})-\frac{3}{2} \partial_{x}^{2} I_{0}(2 \sqrt{x})\right.\right. \\
& \left.-\frac{1}{2} \partial_{x}^{3} I_{0}(2 \sqrt{x})\right)+\zeta_{2}\left(\frac{3}{2} I_{0}(2 \sqrt{x})-\frac{1}{2} \partial_{x} I_{0}(2 \sqrt{x})\right. \\
& \left.\left.-\frac{1}{2} \partial_{x}^{2} I_{0}(2 \sqrt{x})+\frac{3}{2} \partial_{x}^{3} I_{0}(2 \sqrt{x})\right)+\zeta_{3} \partial_{x}^{2} I_{0}(2 \sqrt{x})\right] .
\end{aligned}
$$

Each of these relations was checked up to ten loops. For DNLLA, our formula agrees with the result of [45], where the result was obtained via an analytic continuation of the Wilson loop OPE, while the formulas for $\mathrm{DN}^{(2,3)} \mathrm{LLA}$ are new predictions. Note that we 
refrain from spelling out the real parts which can be obtained from the imaginary parts, as explained before. In eq. (5.4) we have chosen to write all relations in terms of $I_{0}(2 \sqrt{x})$ and derivatives thereof. Whether a simpler form can be found by using the numerous identities among the functions $I_{n}(2 \sqrt{x})$ is left as an open question for future research.

\section{Proof of Pennington's formula}

In [23] Pennington put forward a simple formula for the remainder function in LLA in momentum space. This formula was stated as a conjecture, based on the available data. In this section we proof Pennington's formula using the techniques explained in the earlier sections.

\subsection{Pennington's formula}

We begin by spelling out the formula for the six-gluon MHV remainder function in LLA as described in ref. [23],

$$
\left.R_{6}^{\mathrm{MHV}}\right|_{\text {LLA }}=\frac{2 \pi i}{\log \left(1-u_{1}\right)}\left(\mathcal{X} \mathcal{Z}^{\mathrm{MHV}}-\frac{1}{2} x_{1} \eta\right)
$$

where $\eta=a \log \left(1-u_{1}\right)$ and

$$
\begin{aligned}
\mathcal{X} & =e^{\frac{1}{2} x_{0} \eta}\left[1-x_{1}\left(\frac{e^{x_{0} \eta}-1}{x_{0}}\right)\right]^{-1}, \\
\mathcal{Z}^{\mathrm{MHV}} & =\frac{1}{2} \sum_{k=1}^{\infty}\left(x_{1} \sum_{n=0}^{k-1}(-1)^{n} x_{0}^{k-n-1} \sum_{m=0}^{n} \frac{2^{2 m-k+1}}{(k-m-1) !} \mathfrak{Z}(n, m)\right) \eta^{k} .
\end{aligned}
$$

The coefficients $\mathfrak{Z}(n, m)$ are generated by the series

$$
\exp \left[y \sum_{k=1}^{\infty} \zeta_{2 k+1} x^{2 k+1}\right]=: \sum_{n=0}^{\infty} \sum_{m=0}^{\infty} \mathfrak{Z}(n, m) x^{n} y^{m} .
$$

The above formula can in principle be expanded to any order in the coupling constant $a$ and was checked to 14 loops in ref. [23].

\subsection{Strategy of the proof}

We will prove eq. (6.1) by direct evaluation of the dispersion relation eq. (2.5). By expanding the dispersion relation eq. (2.5) it is easy to see that the $\ell$-loop contribution in LLA is given by

$$
g_{\ell-1}^{(\ell)}=\frac{1}{4(\ell-1) !} \mathcal{I}\left[\left(E^{(0)}\right)^{\ell-1}\right]
$$

while the real part vanishes in LLA, $h_{\ell-1}^{(\ell)}=0$. Using relation (3.4) the $\ell$-loop contribution to the remainder function can be written as

$$
g_{\ell-1}^{(\ell)}=\frac{1}{4(\ell-1) !} \sum_{k=0}^{\ell-1}\left(\begin{array}{c}
\ell-1 \\
k
\end{array}\right)\left(-\frac{1}{2}\right)^{k} \sum_{m=0}^{k}(-1)^{k-m}\left(\begin{array}{c}
k \\
m
\end{array}\right) \mathcal{I}\left[\chi_{+}^{m} \chi_{-}^{k-m} E_{\psi}^{\ell-1-k}\right] .
$$


As described in section 3 we understand the insertion of the factors $\chi_{ \pm}$in the integrand, so we only need to calculate the basis integrals $\mathcal{I}\left[E_{\psi}^{k}\right]$. These were already considered in eq. (3.23) and we can directly rewrite them in HPLs,

$$
\begin{aligned}
\mathcal{I}_{\bullet}\left[E_{\psi}^{k}\right] & =(-2 i) \sum_{n=1}^{\infty} \frac{(-w)^{n}}{-i n} Z_{1}(n)^{k}=2 \sum_{n=1}^{\infty} \frac{(-w)^{n}}{n} \sum_{l=1}^{k} \sum_{\alpha \in T(l, k)} \frac{k !}{\prod \alpha_{i} !} Z_{\alpha_{1}, \ldots, \alpha_{l}}(n) \\
& =2 \sum_{l=1}^{k} \sum_{\alpha \in T(l, k)} \frac{k !}{\prod \alpha_{i} !}\left(H_{1, \alpha_{1}, \ldots, \alpha_{l}}(-w)+H_{\alpha_{1}+1, \ldots, \alpha_{l}}(-w)\right),
\end{aligned}
$$

where the first step follows from a simple inductive proof and the second step uses the definition of the harmonic polylogarithms in terms of $Z$-sums (3.24), as well as relation (3.25). Furthermore, $T(l, k)$ is the set of $l$-tuples of non-negative integers such that their sum is $k$. Note that the result is written in the collapsed notation for HPLs. This allows us to immediately write down the full result via the replacement rule (3.3),

$$
\mathcal{I}\left[E_{\psi}^{k}\right]=2 \sum_{l=1}^{k} \sum_{\alpha \in T(l, k)} \frac{k !}{\prod \alpha_{i} !}\left(\mathcal{L}_{1, \alpha_{1}, \ldots, \alpha_{l}}+\mathcal{L}_{\alpha_{1}+1, \ldots, \alpha_{l}}\right)
$$

where we have used that the residues at $\nu=n=0$ vanish for these basis integrals, see eq. (3.28). Together with the result for $k=0$, eq. (3.9), we have determined all necessary integrals. The proof now proceeds as follows. In the next section, we will first prove eq. (6.1) for the $\zeta$-free part, for which the insertion of $\chi_{ \pm}$is well understood. In section 6.4 we will then examine the appearance of $\zeta$-values and show that for any combination of $\zeta$ 's the direct calculation reproduces Pennington's result.

\subsection{Proof of the $\zeta$-free part}

As described in section 3 inserting factors $\chi_{ \pm}$in the integrand simply appends or prepends factors of $\left(-x_{0}\right)$, neglecting potential $\zeta$ 's for the moment. We can then immediately write down the full expression for the remainder function as

$$
\begin{aligned}
& \left.\frac{1}{2 \pi i} \log \left(1-u_{1}\right) R_{6}^{\mathrm{MHV}}\right|_{\zeta-\text { free }}=\sum_{\ell=1}^{\infty} \eta^{\ell} g_{\ell-1}^{(\ell)}-\eta g_{0}^{(1)} \\
& \quad=\sum_{\ell=1}^{\infty} \sum_{k=0}^{\ell-1} \sum_{m=0}^{k} \frac{(-1)^{m}}{2^{k}} \frac{\eta^{\ell}}{4(\ell-1) !}\left(\begin{array}{c}
\ell-1 \\
k
\end{array}\right)\left(\begin{array}{c}
k \\
m
\end{array}\right) \mathcal{I}\left[\chi_{+}^{m} \chi_{-}^{k-m} E_{\psi}^{\ell-1-k}\right]-\frac{\eta}{4}\left(x_{0}+2 x_{1}\right) \\
& =\cdots=\sum_{m=0}^{\infty} \sum_{k=0}^{\infty} \sum_{\ell=0}^{\infty} \frac{(-1)^{m}}{2^{k+m}} \frac{\eta^{\ell+k+m+1}}{4 \ell ! m ! k !} \mathcal{I}\left[\chi_{+}^{m} \chi_{-}^{k} E_{\psi}^{\ell}\right]-\frac{\eta}{4}\left(x_{0}+2 x_{1}\right) \\
& =\frac{\eta}{4} e^{-\frac{\eta}{2} x_{0}}\left(\sum_{\ell=0}^{\infty} \frac{\eta^{\ell}}{\ell !} \mathcal{I}\left[E_{\psi}^{\ell}\right]\right) e^{\frac{\eta}{2} x_{0}}-\frac{\eta}{4}\left(x_{0}+2 x_{1}\right)
\end{aligned}
$$

where in the intermediate steps we have only relabeled variables and switched orders of summation, which is tedious but straightforward. The last expression in eq. (6.11) is 
already nicely factorized, we only have to rewrite the factor

$$
\sum_{\ell=0}^{\infty} \frac{\eta^{\ell}}{\ell !} \mathcal{I}\left[E_{\psi}^{\ell}\right]
$$

whose summands we have already determined in eq. (6.9). To find a simpler representation let us write down the coefficient of an arbitrary SVHPL $c_{n_{1}, \ldots, n_{m}} \mathcal{L}_{n_{1}, \ldots, n_{m}}$ (in collapsed notation) appearing in eq. (6.9) which is given by

$$
c_{n_{1}, \ldots, n_{m}}=\frac{2}{\eta} \frac{\eta^{\sum n_{i}}}{\left(n_{1}-1\right) ! n_{2} ! \cdots n_{m} !},
$$

as is easily checked. ${ }^{7}$ This, however, is exactly the coefficient one obtains for the expansion of the compact expression

$$
\sum_{\ell=0}^{\infty} \frac{\eta^{\ell}}{\ell !} \mathcal{I}\left[E_{\psi}^{\ell}\right]=2 e^{x_{0} \eta}\left[1-x_{1}\left(\frac{e^{x_{0} \eta}-1}{x_{0}}\right)\right]^{-1} x_{1}+x_{0}
$$

where the last $x_{0}$ arises from the $n=0$ residue of $\mathcal{I}[1]$. Plugging this result in the remainder function eq. (6.11) we obtain the expression written down in [23].

\subsection{Including the $\zeta$ 's}

Let us now include the $\zeta$ 's. To do so, we will determine the resummed remainder function for an arbitrary combination of $\zeta$ 's and show the equivalence with the result of [23]. Since the basis integrals $\mathcal{I}\left[E_{\psi}^{k}\right]$ never contain any $\zeta$ 's by themselves, $\zeta$-values are only created through insertions of $\chi_{+}, \chi_{-} \cdot{ }^{8}$ We are therefore led to consider the integrals

$$
\mathcal{I}_{\bullet}\left[\chi_{+}^{m} \chi_{-}^{k} E_{\psi}^{\ell}\right]+\mathcal{I}_{0}\left[\chi_{+}^{m} \chi_{-}^{k} E_{\psi}^{\ell}\right]
$$

which we will abbreviate as $\mathcal{I}_{\bullet+0}\left[\chi_{+}^{m} \chi_{-}^{k} E_{\psi}^{\ell}\right]$. Let us first consider the case of a single $\zeta$ as a warm-up. By directly evaluating the residues we find

$$
\begin{aligned}
\mathcal{I}_{\bullet+0}\left[\chi_{-}^{k} \chi_{+}^{m} E_{\psi}^{\ell}\right]_{\zeta_{a+1}}= & \left.(-2 i) i^{m+k} \sum_{n=1}^{\infty}(-1)^{n} \frac{i^{m}}{m !} D_{\nu}^{m}\left(\frac{w^{i \nu+\frac{n}{2}}}{\left(\nu-i \frac{n}{2}\right)^{k+1}} E_{\psi}^{\ell}\right)\right|_{\nu=-i \frac{n}{2}} \\
& +\left.(-i) i^{m+k} \frac{i^{m+k+1}}{(m+k+1) !} D_{\nu}^{m+k+1}\left(w^{i \nu} E_{\psi}^{\ell}\right)\right|_{\nu=0} \\
= & i^{a} \ell \frac{\left.\left(D_{\nu}^{a} E_{\psi}\right)\right|_{\nu=-i \frac{n}{2}}}{a !} \mathcal{I}_{\bullet}\left[\chi_{-}^{k} \chi_{+}^{m-a} E_{\psi}^{\ell-1}\right]_{\zeta-\text { free }} \\
& +i^{a} \ell \frac{\left.\left(D_{\nu}^{a} E_{\psi}\right)\right|_{\nu=0}}{a !} \mathcal{I}_{0}\left[\chi_{-}^{k} \chi_{+}^{m-a} E_{\psi}^{\ell-1}\right]_{\zeta \text { free }} \\
= & (-2 \ell) \zeta_{a+1} \mathcal{I}_{\bullet+0}\left[\chi_{-}^{k} \chi_{+}^{m-a} E_{\psi}^{\ell-1}\right]_{\zeta-\text { free }} .
\end{aligned}
$$

\footnotetext{
${ }^{7}$ The simplest way to obtain eq. (6.13) is to see that in eq. (6.9) $\ell$ determines the number of 1 s, while $k$ determines the length of the SVHPL. Writing down the two possible cases for $n_{1}=1, n_{1}>1$ immediately leads to our expression.

${ }^{8}$ Here we see another great benefit of working on the level of HPLs using the replacement $H \rightarrow \mathcal{L}$ : any term containing $\zeta$ 's will explicitly appear in the final answer, while in the original formulation $\zeta$-terms can potentially be absorbed into the definition of SVHPLs.
} 
In the resulting expression we project the remaining integral to the $\zeta$-free part because we are only interested in the piece proportional to $\zeta_{a+1}$, the neglected terms do, however, contribute to other $\zeta$-structures. Similarly, in going from the second to third line in eq. (6.16) we have neglected a Euler sum in the derivative $\left.\left(D_{\nu}^{a} E_{\psi}\right)\right|_{\nu=-i \frac{n}{2}}$, which does not contain any $\zeta$ 's. Note that the next-to-last step in eq. (6.16) also shows that only odd $\zeta$ 's can appear, since even $\zeta$ 's never appear in the derivatives of $E_{\psi}$ (cf. eqs. (3.19) and (3.20)). The step to an arbitrary combination of $\zeta$ 's is now straightforward, we only have to be careful about combinatorial factors. We find

$$
\mathcal{I}_{\bullet+0}\left[\chi_{-}^{k} \chi_{+}^{m} E_{\psi}^{\ell}\right]_{\prod_{i} \zeta_{a_{i}+1}}=(-2)^{n_{z}} \frac{\ell !}{\left(\ell-n_{z}\right) !} \frac{\prod_{i}^{n_{z}} \zeta_{a_{i}+1}}{\prod_{a_{i}} n_{a_{i}} !} \mathcal{I}_{\bullet+0}\left[\chi_{-}^{k} \chi_{+}^{m-\sum a_{i}} E_{\psi}^{\ell-n_{z}}\right]_{\zeta-\text { free }}
$$

where $n_{z}$ is the total number of $\zeta$ 's and $n_{a_{i}}$ counts the multiplicity of $\zeta_{a_{i}+1}$. Using this result, we can immediately calculate the remainder function proportional to this $\zeta$-structure starting from eq. (6.10). For an arbitrary product of $\zeta$ 's, $\prod_{i}^{n_{z}} \zeta_{a_{i}+1}$, we need $\ell \geq n_{z}, m \geq$ $\sum_{i} a_{i}$, so we obtain

$$
\begin{aligned}
& \left.\frac{1}{2 \pi i} \log \left(1-u_{1}\right) R_{6}^{\mathrm{MHV}}\right|_{\prod \zeta_{a_{i}+1}} \\
& \quad=\sum_{\ell=n_{z}}^{\infty} \sum_{m=\sum a_{i}}^{\infty} \sum_{k=0}^{\infty}(-1)^{m} \frac{\eta^{\ell+m+k+1}}{4} \frac{2^{-k-m}}{\ell ! k ! m !} \mathcal{I}\left[\chi_{+}^{m} \chi_{-}^{k} E_{\psi}^{\ell}\right]_{\prod \zeta_{a_{i}+1}} \\
& =\sum_{\ell=n_{z}}^{\infty} \sum_{m=\sum}^{\infty} \sum_{a_{i}}^{\infty} \frac{\eta^{\ell+m+k+1}}{4} \frac{(-1)^{m} 2^{-k-m}}{\left(\ell-n_{z}\right) ! k ! m !}(-2)^{n_{z}} \frac{\prod_{i} \zeta_{a_{i}+1}}{\prod_{a_{i}} n_{a_{i}} !} \mathcal{I}\left[\chi_{+}^{m-\sum a_{i}} \chi_{-}^{k} E_{\psi}^{\ell-n_{z}}\right]_{\zeta-\text { free }} \\
& \quad=\cdots=(-1)^{w} \eta^{w+1} 2^{2 n_{z}-w} \frac{\prod_{i} \zeta_{a_{i}+1}}{\prod_{a_{i}} n_{a_{i}} !} \frac{1}{2} \mathcal{X} x_{1} \sum_{k=0}^{\infty}\left(\frac{\eta}{2}\right)^{k} \frac{x_{0}^{k}}{\left(k+w-n_{z}\right) !}
\end{aligned}
$$

where we used eq. (6.17) in the second step and in the last step we used that the weight of our $\zeta$-structure is given by $w=\sum_{i} a_{i}+n_{z}$. To compare results, we make use of an alternative representation of $\mathfrak{Z}(n, m)$ as given in [23],

$$
\mathfrak{Z}(n, m)=\sum_{\beta \in P(n, m)} \prod_{i} \frac{\left(\zeta_{2 i+1}\right)^{\beta_{i}}}{\beta_{i} !}
$$

where $P(n, m)$ runs over the set of $n$-tuples of non-negative integers with sum $m$ such that the weight of the product of $\zeta$ 's is given by $n$. This means that the remainder function multiplying a given combination of $\zeta$ 's of weight $w$ and consisting of $n_{z} \zeta$ 's as obtained 
from eq. (6.1) is given by

$$
\begin{aligned}
\left.\frac{1}{2 \pi i} \log \left(1-u_{1}\right) R_{6}^{\mathrm{MHV}}\right|_{\mathrm{LLA}, \prod_{i}\left(\zeta_{2 i+1}\right)^{\beta_{i}}} & \\
= & \frac{1}{2} \mathcal{X} \sum_{k=w+1} x_{1}(-1)^{w} x_{0}^{k-w-1} \frac{2^{2 n_{z}-k+1}}{\left(k-n_{z}-1\right) !}\left(\left.\mathfrak{J}\left(w, n_{z}\right)\right|_{\prod\left(\zeta_{2 i+1}\right)^{\beta_{i}}}\right) \eta^{k} \\
& =(-1)^{w} \eta^{w+1} 2^{2 n_{z}-w}\left(\prod_{i} \frac{\left(\zeta_{2 i+1}\right)^{\beta_{i}}}{\beta_{i} !}\right) \frac{1}{2} \mathcal{X} x_{1} \sum_{k=0}^{\infty}\left(\frac{\eta}{2}\right)^{k} \frac{x_{0}^{k}}{\left(k+w-n_{z}\right) !} .
\end{aligned}
$$

This exactly matches our result eq. (6.18), which finishes the proof.

A similar formula for the NMHV case was put also forward in [23]. As is shown there, this NMHV formula satisfies the differential equation

$$
\left.w^{*} \frac{\partial}{\partial w^{*}} R_{6}^{\mathrm{MHV}}\right|_{\mathrm{LLA}}=\left.w \frac{\partial}{\partial w} R_{6}^{\mathrm{NMHV}}\right|_{\mathrm{LLA}},
$$

relating the MHV and NMHV formulas (see also eq. (4.5)). Together with our proof of the MHV formula, this also establishes the NMHV formula presented in [23], although a derivation directly from the dispersion relation eq. (4.2) following the method above should also be possible.

\section{Conclusions}

In this paper, we have presented an efficient algorithm for the calculation of the six-point remainder function of planar $\mathcal{N}=4$ super-Yang-Mills theory in the multi-Regge regime. So far, this calculation had to be carried out by performing an inverse Fourier-Mellin transform by either direct calculation or by making a suitable ansatz and matching series expansions, which becomes involved for a large number of loops or a high logarithmic order. The key improvement which our algorithm provides is that it works directly in momentum space, without the need to make an ansatz or to carry out any non-trivial integrals. It is built on the observation made in ref. [35] that the full result is already encoded in a small subset of the residues appearing in the Fourier-Mellin transform, working under the assumption that the full result will be expressed through SVHPLs. We use this observation to derive recursion relations between different integrals, which allow to reduce any integral appearing in the expansion of the remainder function to a small set of basis integrals. These, in turn, can be trivially evaluated in terms of Euler $Z$-sums. The full result is then obtained from the basis integrals by simply performing the natural actions on a SVHPL, namely attaching an index and shuffling. Using these results, we are able to prove the formula for the MHV remainder function in LLA conjectured in ref. [23]. Our algorithm can be combined with expressions for the BFKL eigenvalue and impact factor in Fourier-Mellin space derived in ref. [29], which are conjectured to be exact, to generate data for any number of loops at any logarithmic order in principle. As specific applications of this, we provide plots for the remainder function in $\mathrm{N}^{3} \mathrm{LLA}$ up to ten loops and for $\mathrm{N}^{4} \mathrm{LLA}$ up to nine loops. The highest number of loops for which we fully determine the remainder function is seven, but 
extending this result to higher loops should be possible. Based on this data, we also write down conjectures for the remainder function in the collinear-Regge regime.

Several directions for future research emerge from our results: it would be interesting to study other contexts in which SVHPLs arise, most notably the application to MuellerNavelet jets [24]. Furthermore, in this paper we have focused on the six-point case. However, similar dispersion relations can be written down in the multi-Regge regime of higherpoint amplitudes, as well $[18,21]$. It would be interesting to understand how our findings carry over to these situations.

\section{Acknowledgments}

We would like to thank Reinke Sven Isermann for collaboration in the early stages of the project. Furthermore, we are grateful to Claude Duhr, Georgios Papathanasiou and Matteo Rosso for helpful discussions and comments on our draft. The research of JB is supported in part by the SFB 647 "Raum-Zeit- Materie. Analytische und Geometrische Strukturen" and the Marie Curie Network GATIS (gatis.desy.eu) of the European Union's Seventh Framework Programme FP7-2007-2013 under grant agreement No. 317089. The work of JB and MS is partially supported by the Swiss National Science Foundation through the NCCR SwissMap.

\section{A Differential equations for insertions of $\chi_{ \pm}$}

In this appendix, we show schematically how to prove that the differential equations eqs. (3.12) and (3.13) hold on the level of the integral and not just on the level of the integrand. We begin by showing

$$
w^{*} \partial_{w^{*}} \mathcal{I}\left[\chi_{+}^{n_{1}} \chi_{-}^{n_{2}} E_{\psi}^{n_{3}}\right]=-\mathcal{I}\left[\chi_{+}^{n_{1}-1} \chi_{-}^{n_{2}} E_{\psi}^{n_{3}}\right] .
$$

Extracting the left-hand side of eq. (A.1) is slightly subtle since our prescription eq. (3.3) is to set $w^{*}=1$ which would naïvely set the left-hand side to zero. Instead we first have to calculate the integral, then act with the differential operator and only then set $w^{*}=1$. For this, it is enough to extract the contribution proportional to $\log w^{*}$, which evaluates to 1 when acted upon with the differential operator, as all other contributions with $w^{*}$-dependence drop out once we set $w^{*}=1$. We find

$$
\begin{aligned}
& w^{*} \partial_{w^{*}} \mathcal{I}_{\bullet}\left[\chi_{+}^{n_{1}} \chi_{-}^{n_{2}} E_{\psi}^{n_{3}}\right] \\
& \quad=\left.\left(w^{*} \partial_{w^{*}}\left((-2 i) \sum_{n=1}^{\infty}(-1)^{n} \frac{(-1)^{n_{1}}}{n_{1} !} i^{n_{2}} D_{\nu}^{n_{1}}\left(\frac{w^{i \nu+\frac{n}{2}}\left(w^{*}\right)^{i \nu-\frac{n}{2}}}{\left(\nu+i \frac{n}{2}\right)^{n_{2}+1}} E_{\psi}^{n_{3}}\right)\right)\right)\right|_{w^{*}=1, \nu=-i \frac{n}{2}} \\
& \quad=\left.(-1)\left((-2 i) \sum_{n=1}^{\infty}(-1)^{n} \frac{(-1)^{n_{1}-1}}{\left(n_{1}-1\right) !} i^{n_{2}} D_{\nu}^{n_{1}-1}\left(\frac{w^{i \nu+\frac{n}{2}}}{\left(\nu+i \frac{n}{2}\right)^{n_{2}+1}} E_{\psi}^{n_{3}}\right)\right)\right|_{\nu=-i \frac{n}{2}} \\
& \quad=-\mathcal{I}_{\bullet}\left[\chi_{+}^{n_{1}-1} \chi_{-}^{n_{2}} E_{\psi}^{n_{3}}\right]
\end{aligned}
$$


Similarly, we have

$$
\begin{aligned}
w^{*} \partial_{w^{*}} \mathcal{I}_{0} & {\left[\chi_{+}^{n_{1}} \chi_{-}^{n_{2}} E_{\psi}^{n_{3}}\right] } \\
& =\left.\left(w^{*} \partial_{w^{*}} \frac{(-1)^{n_{1}+n_{2}}}{\left(n_{1}+n_{2}+1\right) !} D_{\nu}^{n_{1}+n_{2}+1}\left(w^{i \nu+\frac{n}{2}}\left(w^{*}\right)^{i \nu-\frac{n}{2}} E_{\psi}^{n_{3}}\right)\right)\right|_{w^{*}=1, \nu=0} \\
& =\left.\frac{(-1)^{n_{1}+n_{2}}}{\left(n_{1}+n_{2}\right) !} D_{\nu}^{n_{1}+n_{2}}\left(w^{i \nu+\frac{n}{2}} E_{\psi}^{n_{3}}\right)\right|_{\nu=0} \\
& =-\mathcal{I}_{0}\left[\chi_{+}^{n_{1}-1} \chi_{-}^{n_{2}} E_{\psi}^{n_{3}}\right]
\end{aligned}
$$

which, using eq. (3.3), finishes the calculation. Showing

$$
w \partial_{w} \mathcal{I}\left[\chi_{+}^{n_{1}} \chi_{-}^{n_{2}} E_{\psi}^{n_{3}}\right]=-\mathcal{I}\left[\chi_{+}^{n_{1}} \chi_{-}^{n_{2}-1} E_{\psi}^{n_{3}}\right]
$$

is slightly more difficult, since although we can set $w^{*}=1$ right away, we have to take into account all contributions from $w$. We begin with the residue at $\nu=n=0$ and obtain

$$
\begin{aligned}
w \partial_{w} \mathcal{I}_{0} & {\left[\chi_{+}^{n_{1}} \chi_{-}^{n_{2}} E_{\psi}^{n_{3}}\right] } \\
& =\left.w \partial_{w}\left((-i) \frac{(-1)^{n_{1}+n_{2}+1}}{\left(n_{1}+n_{2}+1\right) !} D_{\nu}^{n_{1}+n_{2}+1}\left(w^{i \nu} E_{\psi}^{n_{2}}\right)\right)\right|_{\nu=0} \\
& =\left.\frac{(-1)^{n_{1}+n_{2}}}{\left(n_{1}+n_{2}+1\right) !} \sum_{k=1}^{n_{1}+n_{2}+1}\left(\begin{array}{c}
n_{1}+n_{2}+1 \\
k
\end{array}\right) k \log ^{k-1} w D_{\nu}^{n_{1}+n_{2}+1-k}\left(E_{\psi}^{n_{3}}\right)\right|_{\nu=0} \\
& =\left.\frac{(-1)^{n_{1}+n_{2}}}{\left(n_{1}+n_{2}\right) !} D_{\nu}^{n_{1}+n_{2}}\left(w^{i \nu} E_{\psi}^{n_{2}}\right)\right|_{\nu=0} \\
& =-\mathcal{I}_{0}\left[\chi_{+}^{n_{1}} \chi_{-}^{n_{2}-1} E_{\psi}^{n_{3}}\right] .
\end{aligned}
$$

Moving on to the other residues we find

$$
\begin{aligned}
\mathcal{I}_{\bullet} & \left.\chi_{+}^{n_{1}} \chi_{-}^{n_{2}-1} E_{\psi}^{n_{3}}\right] \\
= & \left.(-2 i) \sum_{n=1}^{\infty}(-1)^{n} \frac{(-1)^{n_{1}}}{n_{1} !} i^{n_{2}-1} D_{\nu}^{n_{1}}\left(\frac{w^{i \nu+\frac{n}{2}}}{\left(\nu-i \frac{n}{2}\right)^{n_{2}}} E_{\psi}^{n_{3}}\right)\right|_{\nu=-i \frac{n}{2}} \\
= & \left.(-2 i) \sum_{n=1}^{\infty}(-1)^{n} \frac{(-1)^{n_{1}}}{n_{1} !} i^{n_{2}-1} D_{\nu}^{n_{1}}\left(\frac{w^{i \nu+\frac{n}{2}}}{\left(\nu-i \frac{n}{2}\right)^{n_{2}+1}} E_{\psi}^{n_{3}}\left(\nu-i \frac{n}{2}\right)\right)\right|_{\nu=-i \frac{n}{2}} \\
= & (-2 i) \sum_{n=1}^{\infty}(-1)^{n} \frac{(-1)^{n_{1}}}{n_{1} !} \\
& \times\left.\left(-n i^{n_{2}} D_{\nu}^{n_{1}}\left(\frac{w^{i \nu+\frac{n}{2}}}{\left(\nu-i \frac{n}{2}\right)^{n_{2}}} E_{\psi}^{n_{3}}\right)+n_{1} i^{n_{2}} D_{\nu}^{n_{1}-1}\left(\frac{w^{i \nu+\frac{n}{2}}}{\left(\nu-i \frac{n}{2}\right)^{n_{2}}} E_{\psi}^{n_{3}}\right)\right)\right|_{\nu=-i \frac{n}{2}} \\
= & -w \partial_{w} \mathcal{I}_{\bullet}\left[\chi_{+}^{n_{1}} \chi_{-}^{n_{2}} E_{\psi}^{n_{3}}\right],
\end{aligned}
$$

where the last step follows from expanding in the number of derivatives acting on $w$ as in eq. (A.5)) This establishes eq. (3.12). 
Open Access. This article is distributed under the terms of the Creative Commons Attribution License (CC-BY 4.0), which permits any use, distribution and reproduction in any medium, provided the original author(s) and source are credited.

\section{References}

[1] L.N. Lipatov, Reggeization of the Vector Meson and the Vacuum Singularity in Nonabelian Gauge Theories, Sov. J. Nucl. Phys. 23 (1976) 338 [inSPIRE].

[2] V.S. Fadin, E.A. Kuraev and L.N. Lipatov, On the Pomeranchuk Singularity in Asymptotically Free Theories, Phys. Lett. B 60 (1975) 50 [inSPIRE].

[3] E.A. Kuraev, L.N. Lipatov and V.S. Fadin, Multi-Reggeon Processes in the Yang-Mills Theory, Sov. Phys. JETP 44 (1976) 443 [INSPIRE].

[4] I.I. Balitsky and L.N. Lipatov, The Pomeranchuk Singularity in Quantum Chromodynamics, Sov. J. Nucl. Phys. 28 (1978) 822 [InSPIRE].

[5] L.N. Lipatov, High-energy asymptotics of multicolor QCD and exactly solvable lattice models, hep-th/9311037 [INSPIRE].

[6] L.N. Lipatov, Asymptotic behavior of multicolor QCD at high energies in connection with exactly solvable spin models, JETP Lett. 59 (1994) 596 [INSPIRE].

[7] L.D. Faddeev and G.P. Korchemsky, High-energy QCD as a completely integrable model, Phys. Lett. B 342 (1995) 311 [hep-th/9404173] [INSPIRE].

[8] Z. Bern, L.J. Dixon and V.A. Smirnov, Iteration of planar amplitudes in maximally supersymmetric Yang-Mills theory at three loops and beyond, Phys. Rev. D 72 (2005) 085001 [hep-th/0505205] [INSPIRE].

[9] J. Bartels, L.N. Lipatov and A. Sabio Vera, $N=4$ supersymmetric Yang-Mills scattering amplitudes at high energies: The Regge cut contribution, Eur. Phys. J. C 65 (2010) 587 [arXiv:0807.0894] [INSPIRE].

[10] J. Bartels, L.N. Lipatov and A. Sabio Vera, BFKL Pomeron, Reggeized gluons and Bern-Dixon-Smirnov amplitudes, Phys. Rev. D 80 (2009) 045002 [arXiv:0802.2065] [INSPIRE].

[11] V. Del Duca, C. Duhr and V.A. Smirnov, An Analytic Result for the Two-Loop Hexagon Wilson Loop in $N=4$ SYM, JHEP 03 (2010) 099 [arXiv:0911.5332] [INSPIRE].

[12] V. Del Duca, C. Duhr and V.A. Smirnov, The Two-Loop Hexagon Wilson Loop in $N=4$ SYM, JHEP 05 (2010) 084 [arXiv: 1003.1702] [INSPIRE].

[13] J. Bartels, J. Kotanski and V. Schomerus, Excited Hexagon Wilson Loops for Strongly Coupled $N=4$ SYM, JHEP 01 (2011) 096 [arXiv:1009.3938] [INSPIRE].

[14] J. Bartels, V. Schomerus and M. Sprenger, Multi-Regge Limit of the n-Gluon Bubble Ansatz, JHEP 11 (2012) 145 [arXiv:1207.4204] [INSPIRE].

[15] J. Bartels, J. Kotanski, V. Schomerus and M. Sprenger, The Excited Hexagon Reloaded, arXiv:1311.1512 [INSPIRE].

[16] J. Bartels, V. Schomerus and M. Sprenger, Heptagon Amplitude in the Multi-Regge Regime, JHEP 10 (2014) 67 [arXiv:1405.3658] [INSPIRE]. 
[17] J. Bartels, V. Schomerus and M. Sprenger, The Bethe roots of Regge cuts in strongly coupled $\mathcal{N}=4$ SYM theory, JHEP 07 (2015) 098 [arXiv: 1411.2594] [INSPIRE].

[18] J. Bartels, A. Kormilitzin, L.N. Lipatov and A. Prygarin, BFKL approach and $2 \rightarrow 5$ maximally helicity violating amplitude in $\mathcal{N}=4$ super-Yang-Mills theory, Phys. Rev. D 86 (2012) 065026 [arXiv:1112.6366] [INSPIRE].

[19] A. Prygarin, M. Spradlin, C. Vergu and A. Volovich, All Two-Loop MHV Amplitudes in Multi-Regge Kinematics From Applied Symbology, Phys. Rev. D 85 (2012) 085019 [arXiv:1112.6365] [INSPIRE].

[20] J. Bartels, A. Kormilitzin and L. Lipatov, Analytic structure of the $n=7$ scattering amplitude in $\mathcal{N}=4 S Y M$ theory in the multi-Regge kinematics: Conformal Regge pole contribution, Phys. Rev. D 89 (2014) 065002 [arXiv:1311.2061] [INSPIRE].

[21] J. Bartels, A. Kormilitzin and L.N. Lipatov, Analytic structure of the $n=7$ scattering amplitude in $\mathcal{N}=4$ theory in multi-Regge kinematics: Conformal Regge cut contribution, Phys. Rev. D 91 (2015) 045005 [arXiv:1411.2294] [INSPIRE].

[22] L.J. Dixon, C. Duhr and J. Pennington, Single-valued harmonic polylogarithms and the multi-Regge limit, JHEP 10 (2012) 074 [arXiv:1207.0186] [INSPIRE].

[23] J. Pennington, The six-point remainder function to all loop orders in the multi-Regge limit, JHEP 01 (2013) 059 [arXiv: 1209.5357] [INSPIRE].

[24] V. Del Duca, L.J. Dixon, C. Duhr and J. Pennington, The BFKL equation, Mueller-Navelet jets and single-valued harmonic polylogarithms, JHEP 02 (2014) 086 [arXiv:1309.6647] [INSPIRE].

[25] L.N. Lipatov and A. Prygarin, BFKL approach and six-particle $M H V$ amplitude in $N=4$ super Yang-Mills, Phys. Rev. D 83 (2011) 125001 [arXiv:1011.2673] [InSPIRE].

[26] V.S. Fadin and L.N. Lipatov, BFKL equation for the adjoint representation of the gauge group in the next-to-leading approximation at $N=4$ SUSY, Phys. Lett. B 706 (2012) 470 [arXiv:1111.0782] [INSPIRE].

[27] L. Lipatov, A. Prygarin and H.J. Schnitzer, The Multi-Regge limit of NMHV Amplitudes in $N=4$ SYM Theory, JHEP 01 (2013) 068 [arXiv:1205.0186] [INSPIRE].

[28] L.J. Dixon, J.M. Drummond, C. Duhr and J. Pennington, The four-loop remainder function and multi-Regge behavior at NNLLA in planar $N=4$ super-Yang-Mills theory, JHEP 06 (2014) 116 [arXiv:1402.3300] [INSPIRE].

[29] B. Basso, S. Caron-Huot and A. Sever, Adjoint BFKL at finite coupling: a short-cut from the collinear limit, JHEP 01 (2015) 027 [arXiv: 1407.3766] [INSPIRE].

[30] B. Basso, A. Sever and P. Vieira, Spacetime and Flux Tube S-Matrices at Finite Coupling for $N=4$ Supersymmetric Yang-Mills Theory, Phys. Rev. Lett. 111 (2013) 091602 [arXiv:1303.1396] [INSPIRE].

[31] B. Basso, A. Sever and P. Vieira, Space-time S-matrix and Flux tube S-matrix II. Extracting and Matching Data, JHEP 01 (2014) 008 [arXiv:1306.2058] [INSPIRE].

[32] B. Basso, A. Sever and P. Vieira, Space-time S-matrix and Flux-tube S-matrix III. The two-particle contributions, JHEP 08 (2014) 085 [arXiv:1402.3307] [INSPIRE].

[33] B. Basso, A. Sever and P. Vieira, Space-time S-matrix and Flux-tube S-matrix IV. Gluons and Fusion, JHEP 09 (2014) 149 [arXiv:1407.1736] [inSPIRE]. 
[34] F.C. Brown, Polylogarithmes multiples uniformes en une variable, Compt. Rendus Math. 338 (2004) 527.

[35] J.M. Drummond and G. Papathanasiou, Hexagon OPE Resummation and Multi-Regge Kinematics, arXiv: 1507.08982 [INSPIRE].

[36] J. Drummond, C. Duhr, B. Eden, P. Heslop, J. Pennington and V.A. Smirnov, Leading singularities and off-shell conformal integrals, JHEP 08 (2013) 133 [arXiv:1303.6909] [INSPIRE].

[37] L.J. Dixon and M. von Hippel, Bootstrapping an NMHV amplitude through three loops, JHEP 10 (2014) 065 [arXiv: 1408.1505] [INSPIRE].

[38] N. Beisert, B. Eden and M. Staudacher, Transcendentality and Crossing, J. Stat. Mech. (2007) P01021 [hep-th/0610251] [INSPIRE].

[39] S. Caron-Huot, When does the gluon reggeize?, JHEP 05 (2015) 093 [arXiv:1309.6521] [INSPIRE].

[40] G. Papathanasiou, Evaluating the six-point remainder function near the collinear limit, Int. J. Mod. Phys. A 29 (2014) 1450154 [arXiv:1406.1123] [inSPIRE].

[41] S. Moch, P. Uwer and S. Weinzierl, Nested sums, expansion of transcendental functions and multiscale multiloop integrals, J. Math. Phys. 43 (2002) 3363 [hep-ph/0110083] [INSPIRE].

[42] L.J. Dixon, M. von Hippel and A.J. McLeod, The four-loop six-gluon NMHV ratio function, JHEP 01 (2016) 053 [arXiv: 1509.08127] [INSPIRE].

[43] C.W. Bauer, A. Frink and R. Kreckel, Introduction to the GiNaC framework for symbolic computation within the C++ programming language, J. Symb. Comput. 33 (2000) 1 [cs/0004015] [INSPIRE].

[44] J. Bartels, L.N. Lipatov and A. Prygarin, Collinear and Regge behavior of $2 \rightarrow 4 M H V$ amplitude in $N=4$ super Yang-Mills theory, arXiv:1104.4709 [INSPIRE].

[45] Y. Hatsuda, Wilson loop OPE, analytic continuation and multi-Regge limit, JHEP 10 (2014) 38 [arXiv: 1404.6506] [INSPIRE]. 